\title{
Six open string disk amplitude in pure spinor superspace
}

\author{
Carlos R. Mafra ${ }^{\mathrm{a}}$, Oliver Schlotterer ${ }^{\mathrm{b}}$, Stephan Stieberger ${ }^{\mathrm{b}, *}$, \\ Dimitrios Tsimpis ${ }^{\mathrm{c}, 1}$ \\ a Max-Planck-Institut für Gravitationsphysik, Albert-Einstein-Institut, 14476 Potsdam, Germany \\ b Max-Planck-Institut für Physik, Werner-Heisenberg-Institut, 80805 München, Germany \\ c Arnold-Sommerfeld-Center for Theoretical Physics, Department für Physik, \\ Ludwig-Maximilians-Universität München, 80333 München, Germany
}

Received 6 December 2010; received in revised form 10 January 2011; accepted 11 January 2011

Available online 14 January 2011

\begin{abstract}
The tree-level amplitude of six massless open strings is computed using the pure spinor formalism. The OPE poles among integrated and unintegrated vertices can be efficiently organized according to the cohomology of pure spinor superspace. The identification and use of these BRST structures and their interplay with the system of equations fulfilled by the generalized Euler integrals allow the full supersymmetric six-point amplitude to be written in compact form. Furthermore, the complete set of extended Bern-Carrasco-Johansson relations are derived from the monodromy properties of the disk world-sheet and explicitly verified for the supersymmetric numerator factors.
\end{abstract}

(c) 2011 Elsevier B.V. All rights reserved.

\section{Introduction}

Elementary particle physics relies on scattering experiments. The physical cross sections, determined by the scattering amplitudes, reflect the properties of underlying interactions. Already at the tree-level, such computations can be quite complicated, especially when a large number of external particles is involved, like in the scattering processes describing multi-jet produc-

\footnotetext{
* Corresponding author.

E-mail addresses: crmafra@aei.mpg.de (C.R. Mafra), olivers@mppmu.mpg.de (O. Schlotterer), stephan.stieberger@mpp.mpg.de (S. Stieberger), dimitrios.tsimpis@1mu.de (D. Tsimpis).

1 Address after November 1st: Département de Physique, Institut de Physique Nucléaire de Lyon, Université Claude Bernard Lyon 1.
} 
tion at hadron colliders. During the last years remarkable progress has been accumulated in our understanding and in our ability to compute scattering amplitudes, both for theoretical and phenomenological purposes, cf. Ref. [1] for a recent account.

Scattering amplitudes in gauge and gravity theories have a remarkably rich yet simple structure, allowing to develop even more powerful tools to understand their behavior. Various relations within or between gravity and gauge theory scattering amplitudes suggest a unification within or between these theories of the sort inherent to string theory, cf. Ref. [2] for a recent review. Some field-theory properties of scattering amplitudes can be easily derived and proven by string theory. One notorious example are the Kawai, Lewellen and Tye (KLT) relations, which express a graviton amplitude as a sum of squares of partial color ordered gluon amplitudes [3]. Another example are the so-called Bern, Carrasco and Johansson (BCJ) relations, which relate various partial color-ordered subamplitudes [4]. These relations have a natural explanation in string theory: they simply are consequences of monodromy properties on the string world-sheet $[5,6]$. Hence, world-sheet symmetries of string amplitudes turn out to have profound impact on the structure of field-theory amplitudes itself. Therefore, the hidden structures and symmetries of superstring disk scattering amplitudes prove to be useful in revealing properties and symmetries of field-theory amplitudes.

Scattering amplitudes are also of considerable theoretical interest in the framework of a full fledged superstring theory. The pure spinor formalism [7,8] has been useful for quantizing the superstring in Ramond-Ramond backgrounds, and has considerably simplified the computation of multi-loop superstring scattering amplitudes [9-18]. Four- and five-point tree-level [19] amplitudes have been computed in pure spinor superspace and cast into compact form ${ }^{2}$ in Refs. [15, 25].

The pure spinor formalism might also be used to describe $D=11$ supergravity and M-theory. The BRST cohomology properties of the pure spinor superspace [26] are useful not only to simplify the string amplitudes $[15,25]$ but also has recently been suggested of allowing field-theory amplitudes to be obtained directly [27,28]. Furthermore, the BRST cohomology sheds light on the structure and organization of the terms in higher-point open superstring amplitudes. Hence, it is of fundamental importance to pursue multi-leg amplitude computations in pure spinor superspace and anticipate their underlying symmetries, e.g. by exploiting their BRST cohomology properties.

In this work we show that the color-ordered open superstring six-point disk amplitude computed with the pure spinor formalism is given by

$$
\begin{aligned}
A(1,2,3,4,5,6)= & \left\langle T_{54} T_{32} T_{16}\right\rangle F_{1}+\left\langle T_{52} T_{43} T_{16}\right\rangle F_{2}+\left\langle T_{53} T_{42} T_{16}\right\rangle F_{3} \\
& +\left\langle T_{123} E_{456}\right\rangle F_{4}+\left\langle T_{324} E_{561}\right\rangle F_{5}+\left\langle T_{435} E_{216}\right\rangle F_{6}+\left\langle T_{325} E_{416}\right\rangle F_{7} \\
& +\left\langle T_{124} E_{356}\right\rangle F_{8}+\left\langle T_{352} E_{416}\right\rangle F_{9}+\left\langle T_{241} E_{356}\right\rangle F_{10}+(1 \leftrightarrow 5,2 \leftrightarrow 4) .
\end{aligned}
$$

The pure spinor bracket $\langle\ldots\rangle$ was defined in [7] and selects the terms proportional to $\left(\lambda \gamma^{m} \theta\right)\left(\lambda \gamma^{n} \theta\right)\left(\lambda \gamma^{p} \theta\right)\left(\theta \gamma_{m n p} \theta\right)$, which is the unique element in the cohomology of the BRST charge at ghost number three. The factors of $F_{i}$ (and their image $F_{i}^{\prime}$ under $1 \leftrightarrow 5,2 \leftrightarrow 4$ ) denote certain combinations of generalized Euler integrals whose momentum expansions obtained

\footnotetext{
2 In the RNS formalism the five-gluon amplitude has been computed in Refs. [20,21], while the six-gluon amplitude has been computed in Refs. [21-24].
} 

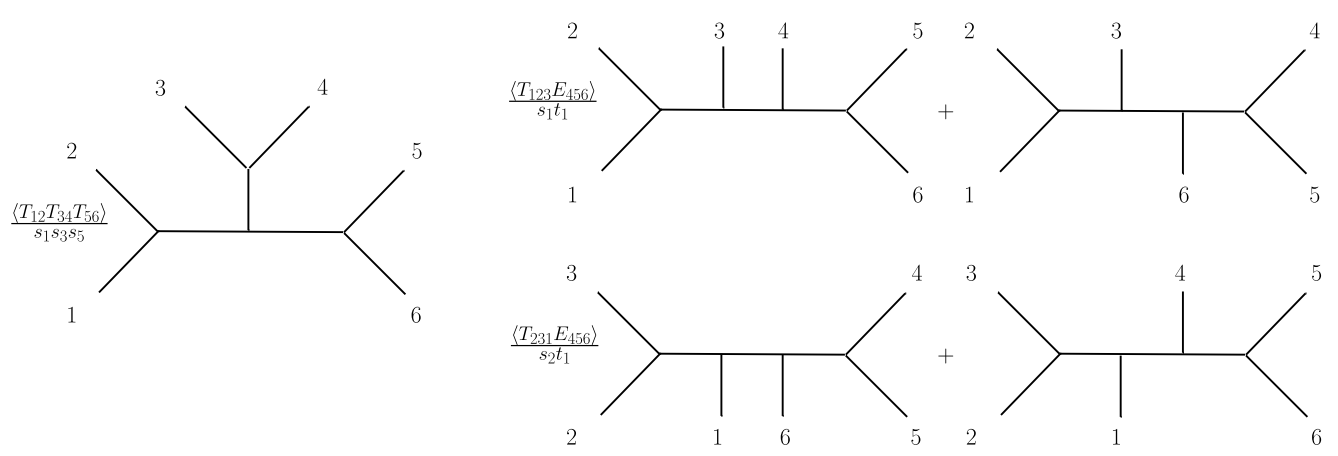

Fig. 1. The five field-theory diagrams and their corresponding pure spinor superspace expressions.

with the methods of [21] are listed in Appendix B. The factors of $\left[T_{i j}, T_{i j k}, E_{i j k}\right]$ are pure spinor BRST building blocks whose definitions and properties will be explained in Section 2. The result (1.1) simplifies and extends the RNS computations of [21] to the full supermultiplet. A compact expression for the full six-point superstring amplitude in the $D=4$ helicity basis can be found in [22-24]. Using the FORM [29] program described in [30], the six-gluon component expansion of (1.1) can be extracted. In fact, up to the order $\alpha^{\prime 3}$, which is available from the authors upon request, we have explicitly verified that the latter agrees with the result of [21]. Furthermore, the field-theory limit $\alpha^{\prime} \rightarrow 0$ of (1.1) is:

$$
\begin{aligned}
A_{\mathrm{SYM}}(1,2,3,4,5,6)= & \frac{\left\langle T_{12} T_{34} T_{56}\right\rangle}{3 s_{1} s_{3} s_{5}}+\frac{1}{2}\left\langle\left(\frac{T_{123}}{s_{1} t_{1}}-\frac{T_{231}}{s_{2} t_{1}}\right) E_{456}\right\rangle+\operatorname{cyclic}(1 \ldots 6) \\
= & \frac{\left\langle T_{12} T_{34} T_{56}\right\rangle}{s_{1} s_{3} s_{5}}+\frac{\left\langle T_{54} T_{32} T_{16}\right\rangle}{s_{2} s_{4} s_{6}}+\frac{\left\langle T_{123} E_{456}\right\rangle}{s_{1} t_{1}}+\frac{\left\langle T_{543} E_{216}\right\rangle}{s_{4} t_{3}} \\
& +\frac{\left\langle T_{321} E_{456}\right\rangle}{s_{2} t_{1}}+\frac{\left\langle T_{345} E_{216}\right\rangle}{s_{3} t_{3}}+\frac{\left\langle T_{432} E_{561}\right\rangle}{s_{3} t_{2}}+\frac{\left\langle T_{234} E_{561}\right\rangle}{s_{2} t_{2}} .
\end{aligned}
$$

The expression (1.2) agrees with the superspace expression recently proposed based on BRST cohomology [27]. The superspace expressions in (1.2) can be interpreted [27] in terms of Feynman diagrams which use only cubic vertices as discussed in [4]. The diagrams associated with the three terms in the first line of (1.2) - which generate the full amplitude upon cyclic symmetrization - are depicted in Fig. 1.

\section{Pure spinor preliminaries}

The prescription to compute the massless open superstring six-point tree-level amplitude is given by [7]

$$
\begin{aligned}
& \mathcal{A}(1,2,3,4,5,6) \\
& \quad=\left\langle V^{1}\left(z_{1}\right) V^{5}\left(z_{5}\right) V^{6}\left(z_{6}\right) \int d z_{2} U^{2}\left(z_{2}\right) \int d z_{3} U^{3}\left(z_{3}\right) \int d z_{4} U^{4}\left(z_{4}\right)\right\rangle,
\end{aligned}
$$

where $V^{i}\left(z_{i}\right)$ and $U^{i}\left(z_{i}\right)$ are the vertex operators with conformal weight zero and one,

$$
V^{i}\left(z_{i}\right)=\lambda^{\alpha}\left(z_{i}\right) A_{\alpha}^{i}\left(X^{i}, \theta\right), \quad U^{i}\left(z_{i}\right)=\partial \theta^{\alpha} A_{\alpha}^{i}+\Pi^{m} A_{m}^{i}+d_{\alpha} W_{i}^{\alpha}+\frac{1}{2} \mathcal{F}_{m n}^{i} N^{m n},
$$


and the positions of the unintegrated vertices are fixed by $S L(2, \mathbf{R})$ invariance to arbitrary locations, which in this paper are chosen as $\left(z_{1}, z_{5}, z_{6}\right)=(0,1, \infty)$. The amplitude (2.1) represents the color ordered subamplitude, given by $\mathcal{A}(1,2,3,4,5,6)=\operatorname{Tr}\left(T^{a_{1}} T^{a_{2}} T^{a_{3}} T^{a_{4}} T^{a_{5}} T^{a_{6}}\right) \times$ $A(1,2,3,4,5,6)$, with the Chan-Paton factors in the adjoint representation.

The operators $\left[d_{\alpha}, \Pi^{m}, N^{m n}, \partial \theta^{\alpha}, \lambda^{\alpha}, w_{\alpha}\right]$ satisfy the OPEs ${ }^{3}$

$$
\begin{aligned}
& d_{\alpha}(z) V(w) \rightarrow \frac{D_{\alpha} V(w)}{z-w}, \quad \Pi^{m}(z) V(w) \rightarrow-\frac{k^{m} V(w)}{z-w}, \\
& d_{\alpha}(z) \Pi^{m}(w) \rightarrow \frac{\left(\gamma^{m} \partial \theta\right)_{\alpha}}{z-w}, \quad d_{\alpha}(z) d_{\beta}(w) \rightarrow-\frac{\gamma_{\alpha \beta}^{m} \Pi_{m}}{z-w}, \\
& \Pi^{m}(z) \Pi^{n}(w) \rightarrow-\frac{\eta^{m n}}{(z-w)^{2}}, \quad d_{\alpha}(z) \theta^{\beta}(w) \rightarrow \frac{\delta_{\alpha}^{\beta}}{(z-w)}, \\
& d_{\alpha}(z) \partial \theta^{\beta}(w) \rightarrow \frac{\delta_{\alpha}^{\beta}}{(z-w)^{2}}, \quad w(z)_{\alpha} \lambda^{\beta}(w) \rightarrow-\frac{\delta_{\alpha}^{\beta}}{z-w}, \\
& N^{m n}(z) \lambda^{\alpha}(w) \rightarrow-\frac{1}{2} \frac{\left(\lambda \gamma^{m n}\right)^{\alpha}}{z-w}, \\
& N^{m n}(z) N_{p q}(w) \rightarrow+\frac{4}{z-w} N_{[p}^{[m} \delta_{q]}^{n]}-\frac{6}{(z-w)^{2}} \delta_{[p}^{n} \delta_{q]}^{m},
\end{aligned}
$$

where $V(w)$ is an arbitrary conformal weight-zero superfield, $N^{m n}=\frac{1}{2}\left(\lambda \gamma^{m n} w\right)$ are the pure spinor Lorentz currents and the antisymmetrization bracket [...] encompassing $N$ indices is defined to contain an overall $1 / N$ !. The super-Yang-Mills superfields $\left[A_{\alpha}, A_{m}, W^{\alpha}, \mathcal{F}_{m n}\right]$ satisfy the equations of motion $[33,31]$

$$
\begin{array}{ll}
D_{\alpha} A_{\beta}+D_{\beta} A_{\alpha}=\gamma_{\alpha \beta}^{m} A_{m}, & D_{\alpha} A_{m}=\left(\gamma_{m} W\right)_{\alpha}+k_{m} A_{\alpha}, \\
D_{\alpha} \mathcal{F}_{m n}=2 k_{[m}\left(\gamma_{n]} W\right)_{\alpha}, & D_{\alpha} W^{\beta}=\frac{1}{4}\left(\gamma^{m n}\right)_{\alpha}^{\beta} \mathcal{F}_{m n},
\end{array}
$$

and have the following $\theta$-expansions in the gauge $\theta^{\alpha} A_{\alpha}=0[34,35]$,

$$
\begin{aligned}
A_{\alpha}(x, \theta)= & \frac{1}{2} a_{m}\left(\gamma^{m} \theta\right)_{\alpha}-\frac{1}{3}\left(\xi \gamma_{m} \theta\right)\left(\gamma^{m} \theta\right)_{\alpha}-\frac{1}{32} F_{m n}\left(\gamma_{p} \theta\right)_{\alpha}\left(\theta \gamma^{m n p} \theta\right)+\cdots, \\
A_{m}(x, \theta)= & a_{m}-\left(\xi \gamma_{m} \theta\right)-\frac{1}{8}\left(\theta \gamma_{m} \gamma^{p q} \theta\right) F_{p q}+\frac{1}{12}\left(\theta \gamma_{m} \gamma^{p q} \theta\right)\left(\partial_{p} \xi \gamma_{q} \theta\right)+\cdots, \\
W^{\alpha}(x, \theta)= & \xi^{\alpha}-\frac{1}{4}\left(\gamma^{m n} \theta\right)^{\alpha} F_{m n}+\frac{1}{4}\left(\gamma^{m n} \theta\right)^{\alpha}\left(\partial_{m} \xi \gamma_{n} \theta\right) \\
& +\frac{1}{48}\left(\gamma^{m n} \theta\right)^{\alpha}\left(\theta \gamma_{n} \gamma^{p q} \theta\right) \partial_{m} F_{p q}+\cdots, \\
\mathcal{F}_{m n}(x, \theta)= & F_{m n}-2\left(\partial_{[m} \xi \gamma_{n]} \theta\right)+\frac{1}{4}\left(\theta \gamma_{[m} \gamma^{p q} \theta\right) \partial_{n]} F_{p q}+\cdots,
\end{aligned}
$$

where $a_{m}(x)=e_{m} \mathrm{e}^{i k \cdot x}, \xi^{\alpha}(x)=\chi^{\alpha} \mathrm{e}^{i k \cdot x}$ are the bosonic and fermionic polarizations and $F_{m n}=$ $2 \partial_{[m} a_{n]}$ is the field strength.

\footnotetext{
3 For reviews of the pure spinor formalism, see $[31,32]$.
} 


\subsection{Six-point kinematic invariants}

By using momentum conservation an $N$-point amplitude can be written in terms of $N(N-3) / 3$ kinematic invariants. It is convenient to define [22]

$$
\begin{aligned}
& s_{i j}=2 \alpha^{\prime} k_{i} \cdot k_{j}, \quad s_{i}=\alpha^{\prime}\left(k_{i}+k_{i+1}\right)^{2}, \quad s_{i j k}=s_{i j}+s_{i k}+s_{j k}, \\
& t_{l}=\alpha^{\prime}\left(k_{l}+k_{l+1}+k_{l+2}\right)^{2}, \quad i, j, k=1, \ldots, 6, l=1,2,3
\end{aligned}
$$

such that all six-point kinematic invariants can be written ${ }^{4}$ in terms of $s_{1}, \ldots, s_{6}$ and $t_{1}, t_{2}, t_{3}$,

$$
\begin{array}{lll}
s_{13}=-s_{1}-s_{2}+t_{1}, & s_{14}=s_{2}+s_{5}-t_{1}-t_{2}, & s_{15}=-s_{5}-s_{6}+t_{2}, \\
s_{24}=-s_{2}-s_{3}+t_{2}, & s_{25}=s_{3}+s_{6}-t_{2}-t_{3}, & s_{26}=-s_{1}-s_{6}+t_{3}, \\
s_{35}=-s_{3}-s_{4}+t_{3}, & s_{36}=s_{1}+s_{4}-t_{1}-t_{3}, & s_{46}=-s_{4}-s_{5}+t_{1} .
\end{array}
$$

The $s_{i}$ and $t_{i}$ variables have well-defined transformations under cyclic transformations; $s_{i+6}=s_{i}$ and $t_{i+3}=t_{i}$. Furthermore, under the worldsheet parity transformation $1 \leftrightarrow 5,2 \leftrightarrow 4$ (also known as twist) the kinematic invariants $s_{i}$ and $t_{i}$ transform as

$$
s_{1} \leftrightarrow s_{4}, \quad s_{2} \leftrightarrow s_{3}, \quad s_{5} \leftrightarrow s_{6}, \quad t_{1} \leftrightarrow t_{3}, \quad t_{2} \leftrightarrow t_{2} .
$$

The six-point amplitude can be shown to be invariant under worldsheet parity, i.e.: $A(1,2,3,4,5,6)=A(6,5,4,3,2,1)$.

\subsection{BRST building blocks}

The simple form of the BRST charge in the pure spinor formalism when acting on superfields, $Q=\lambda^{\alpha} D_{\alpha}$, turns out to allow an efficient method to organize the computations. Inspired by the explicit superspace computations of the four- and five-point amplitudes in [15,25], this method to handle the computations efficiently consists in identifying the so-called BRST building blocks which transform covariantly under the pure spinor BRST charge.

Using this BRST-covariant organization together with the kinematic pole expansion of the tree-level amplitudes discussed in [4], Ansätze for the six- and seven-point super-Yang-Mills amplitudes were presented in [27]. In this section more BRST building blocks which appear naturally in the full superstring six-point amplitude will be identified, expanding and improving the set used in [27].

The OPEs can be used to define $L_{i j}, L_{j i k i}$ and $L_{j i k i l i}$ as

$$
\begin{aligned}
& V^{i}\left(z_{i}\right) U^{j}\left(z_{j}\right) \rightarrow \frac{L_{i j}}{z_{i j}}, \quad L_{i j}\left(z_{i}\right) U^{k}\left(z_{k}\right) \rightarrow \frac{L_{j i k i}}{z_{i k}}, \\
& L_{j i k i}\left(z_{i}\right) U^{l}\left(z_{l}\right) \rightarrow \frac{L_{j i k i l i}}{z_{l i}} .
\end{aligned}
$$

Their explicit expressions are written in Appendix A. It is also convenient to define

$$
\begin{aligned}
& D_{i j}=\left(A^{i} \cdot A^{j}\right), \quad R_{i j k}=D_{i j}\left(A^{k} \cdot\left(k^{i}+k^{j}\right)\right), \\
& O_{i j k}=\frac{1}{2} D_{i j}\left(k^{i} \cdot A^{k}\right)+\text { antisymmetrization in } i, j, k,
\end{aligned}
$$

which are motivated by the residues of $U^{i}\left(z_{i}\right) U^{j}\left(z_{j}\right)$ and $U^{i}\left(z_{i}\right) U^{j}\left(z_{j}\right) U^{k}\left(z_{k}\right)$ double poles.

\footnotetext{
4 From now on we set $\alpha^{\prime}=1 / 2$ unless otherwise stated.
} 
Computing the BRST variations of (2.8) one gets [27],

$$
\begin{aligned}
Q L_{i j}=- & s_{i j} V_{i} V_{j}, \\
Q L_{j i k i}= & s_{i j}\left[L_{j k} V_{i}-L_{i k} V_{j}+L_{i j} V_{k}\right]-s_{i j k} L_{i j} V_{k}, \\
Q L_{j i k i l i}= & \left(k_{i} \cdot k_{j}\right)\left(V_{i} L_{k j l j}+L_{k i l i} V_{j}\right)+\left[\left(k_{i}+k_{j}\right) \cdot k_{k}\right] L_{j i l i} V_{k} \\
& +\left[\left(k_{i}+k_{j}+k_{k}\right) \cdot k_{l}\right] L_{j i k i} V_{l}+\left(k_{i} \cdot k_{j}\right)\left[L_{i k} L_{j l}+L_{i l} L_{j k}\right] \\
& +\left[\left(k_{i}+k_{j}\right) \cdot k_{k}\right] L_{i j} L_{k l} .
\end{aligned}
$$

Using the SYM equations of motion it is easy to see that the symmetric piece of $L_{i j}$ is BRSTexact,

$$
Q D_{i j}=L_{i j}+L_{j i}
$$

which suggests defining the antisymmetric part to be the first composite BRST building block $T_{i j}$

$$
T_{i j} \equiv L_{i j}-\frac{1}{2} Q D_{i j}, \quad Q T_{i j}=-s_{i j} V_{i} V_{j}
$$

Removing the BRST-exact part of $L_{i j}$ in the RHS of (2.12) leads to the definition

$$
\tilde{T}_{i j k} \equiv L_{j i k i}-\frac{s_{i j}}{2}\left[D_{j k} V_{i}-D_{i k} V_{j}+D_{i j} V_{k}\right]+\frac{s_{i j k}}{2} D_{i j} V_{k}
$$

Its BRST variation $Q \tilde{T}_{i j k}=s_{i j} T_{\{i j} V_{k\}}-s_{i j k} T_{i j} V_{k}$ is written in terms of $T_{i j}$ instead of $L_{i j}$, where $\{\ldots\}$ denotes the cyclic symmetrization of the enclosed indices. Note that $\tilde{T}_{(i j) k}$ is BRST-exact

$$
Q R_{i j k}=\tilde{T}_{i j k}+\tilde{T}_{j i k},
$$

and therefore we extract the antisymmetric $[i j]$ part of $\tilde{T}_{i j k}$ as

$$
\tilde{T}_{[i j] k} \equiv \tilde{T}_{i j k}-\frac{1}{2} Q R_{i j k} .
$$

Finally, using the definitions (2.10) and (2.18) it is possible to show that $O_{i j k}$ obeys

$$
Q O_{i j k}=-\tilde{T}_{[i j] k}-\tilde{T}_{[k i] j}-\tilde{T}_{[j k] i}
$$

which finally suggests the definition of the next building block $T_{i j k}$,

$$
T_{i j k} \equiv \tilde{T}_{[i j] k}+\frac{1}{3} Q O_{i j k}=\frac{2}{3} \tilde{T}_{[i j] k}-\frac{1}{3} \tilde{T}_{[k i] j}-\frac{1}{3} \tilde{T}_{[j k] i},
$$

which satisfies

$$
Q T_{i j k}=s_{i j} T_{\{i j} V_{k\}}-s_{i j k} T_{i j} V_{k} .
$$

Note from (2.20) that $T_{i j k}$ has the symmetries of a $(2,1)$-hook,

$$
T_{i j k}=T_{[i j] k}, \quad T_{\{i j k\}}=0 .
$$

It is also convenient to define

$$
E_{i j k} \equiv \frac{V_{i} T_{j k}}{s_{j k}}+\frac{T_{i j} V_{k}}{s_{i j}}
$$


which is BRST-exact,

$$
E_{i j k}=-\frac{1}{s_{i j k}} Q\left(\frac{T_{i j k}}{s_{i j}}+\frac{T_{k j i}}{s_{k j}}\right), \quad Q E_{i j k}=0 .
$$

Following the same BRST reasoning, the next building block $\tilde{T}_{i j k l}$ is defined by

$$
\begin{aligned}
\tilde{T}_{i j k l} \equiv & L_{j i k i l i} \\
& -\frac{1}{2}\left[\left(s_{i j k}-s_{i j}\right)\left(T_{k l} D_{i j}-T_{i j} D_{k l}\right)+s_{i j}\left(T_{j k} D_{i l}+T_{j l} D_{i k}-T_{i k} D_{j l}-T_{i l} D_{j k}\right)\right] \\
& -\frac{1}{4}\left[s_{i j}\left(D_{i k}\left(L_{j l}+L_{l j}\right)+D_{i l}\left(L_{j k}+L_{k j}\right)\right)-\left(s_{i j}-s_{i j k}\right) D_{i j}\left(L_{k l}+L_{l k}\right)\right] \\
& -\frac{1}{2}\left[s_{i j k l} R_{i j k} V_{l}+s_{i j k}\left(R_{i j l} V_{k}-R_{i j k} V_{l}\right)-s_{i j}\left(R_{j k l} V_{i}-R_{i k l} V_{j}+R_{i j l} V_{k}\right)\right] \\
& +\frac{1}{3}\left[s_{i j k l} O_{i j k} V_{l}+s_{i j k}\left(O_{i j l} V_{k}-O_{i j k} V_{l}\right)-s_{i j}\left(O_{j k l} V_{i}-O_{i k l} V_{j}+O_{i j l} V_{k}\right)\right],
\end{aligned}
$$

which satisfies

$$
Q \tilde{T}_{i j k l}=s_{i j k l} T_{i j k} V_{l}+s_{i j k}\left(T_{i j l} V_{k}-T_{i j k} V_{l}+T_{i j} T_{k l}\right)+s_{i j}\left(V_{\{i} T_{j k\} l}-T_{\{i j} T_{k\} l}\right) .
$$

The corrections containing $D_{i j}$ in the second and third lines of (2.25) are required to make the BRST transformation $Q T_{i j k l}$ be written in terms of $T_{i j}$ and $\tilde{T}_{i j k}$ rather than $L_{i j}$ and $L_{j i k i}$. Analogously, the $R_{i j k}$ corrections in the fourth line are needed to further rewrite $\tilde{T}_{i j k}$ in terms of $\tilde{T}_{[i j] k}$ and finally, the $O_{i j k}$ corrections in the fifth line of (2.25) allow $\tilde{T}_{[i j] k}$ to be rewritten in terms of the building block $T_{i j k}$ of (2.20). Therefore the RHS of the BRST variation (2.26) is composed only out of building blocks.

Using (2.26) one can show that

$$
Q \tilde{T}_{(i j) k l}=Q \tilde{T}_{[i j k] l}=Q\left(\tilde{T}_{i j[k l]}+\tilde{T}_{k l[i j]}\right)=0
$$

and we expect that all these combinations are in fact BRST-exact. For example,

$$
\tilde{T}_{i j k l}+\tilde{T}_{j i k l}=Q R_{i j k l}
$$

where

$$
R_{i j k l}=-R_{i j k}\left[\left(k^{i}+k^{j}+k^{k}\right) \cdot A^{l}\right]-\frac{1}{4} s_{i j}\left(D_{i k} D_{j l}+D_{j k} D_{i l}\right) .
$$

Appropriate redefinitions $T_{i j k l}=\tilde{T}_{i j k l}+Q(\ldots)$ lead to a building block with four legs which obeys the symmetry properties (2.27) by itself without $Q$ action:

$$
T_{(i j) k l}=T_{[i j k] l}=T_{i j[k l]}+T_{k l[i j]}=0 .
$$

Since $\tilde{T}_{i j k l}$ enters the six-point amplitude in the combination $\left\langle\tilde{T}_{i j k l} V_{m} V_{n}\right\rangle=\frac{-1}{s_{m n}}\left\langle\tilde{T}_{i j k l} Q T_{m n}\right\rangle$ only, the BRST exact parts decouple and we can replace $\tilde{T}_{i j k l} \leftrightarrow T_{i j k l}$ in all instances throughout this work. 


\section{The six-point amplitude in pure spinor superspace}

With the conventions of the previous section, the following open-string six-point subamplitude will be computed

$$
\begin{aligned}
\mathcal{A} & (1,2,3,4,5,6) \\
& =\left\langle V^{1}\left(z_{1}\right) V^{5}\left(z_{5}\right) V^{6}\left(z_{6}\right) \int d z_{2} U^{2}\left(z_{2}\right) \int d z_{3} U^{3}\left(z_{3}\right) \int d z_{4} U^{4}\left(z_{4}\right)\right\rangle .
\end{aligned}
$$

It would be reasonable to expect that the full explicit computation of this correlator becomes a rather big and tedious expression to work with. However, we will show there are some simplifying features of the pure spinor formalism which allow for an efficient evaluation of (3.1) leading to a simple and compact result written in pure spinor superspace.

To achieve this simplification, we exploit the interplay between kinematic factors in pure spinor superspace and their associated integrals. They both obey different sets of identities which, when considered together, lead to many cancellations at the superspace level.

Identities among the kinematic factors arise from amplitude's independence on the order in which the conformal weight-one variables are integrated out [25]. As will become apparent below, an early application of this method reduces the amount of explicit superfield manipulations considerably. Furthermore, the pure spinor computations are best organized using the BRST building-blocks of the previous section, which has the additional benefit of reusing elements from amplitudes with a lower number of legs.

It is convenient to organize the six-point subamplitude in terms of all possible OPE contractions of the integrated vertex operators. Each OPE contribution is associated with its specific kinematic factor and $z_{i j}$ dependent denominator for the worldsheet integration, as in the fivepoint amplitude of [25]. Using the OPE's to eliminate the conformal weight-one variables with positions (234) and setting $\alpha^{\prime}=1 / 2$, the subamplitude $A(1,2,3,4,5,6)$ is written as the sum of 24 single- and ten double-pole integrals ${ }^{5}$

$$
\begin{aligned}
A(1,2,3,4,5,6) & =\int d z_{2} \int d z_{3} \int d z_{4} \prod_{i<j}^{6}\left|z_{i j}\right|^{-s_{i j}} \\
& \times\left\{\frac{\left\langle L_{213141} V_{5} V_{6}\right\rangle}{z_{21} z_{31} z_{41}}+\frac{\left\langle L_{213145} V_{6}\right\rangle}{z_{21} z_{31} z_{45}}+\frac{\left\langle L_{213541} V_{6}\right\rangle}{z_{21} z_{35} z_{41}}+\frac{\left\langle L_{213545} V_{6}\right\rangle}{z_{21} z_{35} z_{45}}+\frac{\left\langle L_{253141} V_{6}\right\rangle}{z_{25} z_{31} z_{41}}\right. \\
& +\frac{\left\langle L_{253145} V_{6}\right\rangle}{z_{25} z_{31} z_{45}}+\frac{\left\langle L_{253541} V_{6}\right\rangle}{z_{25} z_{35} z_{41}}+\frac{\left\langle L_{253545} V_{1} V_{6}\right\rangle}{z_{25} z_{35} z_{45}}+\frac{\left\langle L_{213441} V_{5} V_{6}\right\rangle}{z_{21} z_{34} z_{41}}+\frac{\left\langle L_{213445} V_{6}\right\rangle}{z_{21} z_{34} z_{45}} \\
& +\frac{\left\langle L_{253441} V_{6}\right\rangle}{z_{25} z_{34} z_{41}}+\frac{\left\langle L_{253445} V_{1} V_{6}\right\rangle}{z_{25} z_{34} z_{45}}+\frac{\left\langle L_{243141} V_{5} V_{6}\right\rangle}{z_{24} z_{31} z_{41}}+\frac{\left\langle L_{243145} V_{6}\right\rangle}{z_{24} z_{31} z_{45}}+\frac{\left\langle L_{243541} V_{6}\right\rangle}{z_{24} z_{35} z_{41}} \\
& +\frac{\left\langle L_{243545} V_{1} V_{6}\right\rangle}{z_{24} z_{35} z_{45}}+\frac{\left\langle L_{233141} V_{5} V_{6}\right\rangle}{z_{23} z_{31} z_{41}}+\frac{\left\langle L_{233541} V_{6}\right\rangle}{z_{23} z_{35} z_{41}}+\frac{\left\langle L_{233145} V_{6}\right\rangle}{z_{23} z_{31} z_{45}} \\
& +\frac{\left\langle L_{233545} V_{1} V_{6}\right\rangle}{z_{23} z_{35} z_{45}}+\frac{\left\langle L_{233441} V_{5} V_{6}\right\rangle}{z_{23} z_{34} z_{41}}+\frac{\left\langle L_{233445} V_{1} V_{6}\right\rangle}{z_{23} z_{34} z_{45}}+\frac{\left\langle L_{243431} V_{5} V_{6}\right\rangle}{z_{24} z_{34} z_{31}}
\end{aligned}
$$

5 Single- or double-pole integral denote the origin of the $z_{i}$ dependence, whether they come from the single or double poles in the OPE's. It is easy to see that the naive number of single-pole integrals in an $N$-point open-string amplitude is $(N-2)$ !. 


$$
\begin{aligned}
& +\frac{\left\langle L_{243435} V_{1} V_{6}\right\rangle}{z_{24} z_{34} z_{35}}+\frac{\left\langle L_{213434} V_{5} V_{6}\right\rangle}{z_{21} z_{34}^{2}}+\frac{\left\langle L_{253434} V_{1} V_{6}\right\rangle}{z_{25} z_{34}^{2}}+\frac{\left\langle L_{242431} V_{5} V_{6}\right\rangle}{z_{24}^{2} z_{31}} \\
& +\frac{\left\langle L_{242435} V_{1} V_{6}\right\rangle}{z_{24}^{2} z_{35}}+\frac{\left\langle L_{232341} V_{5} V_{6}\right\rangle}{z_{23}^{2} z_{41}}+\frac{\left\langle L_{232345} V_{1} V_{6}\right\rangle}{z_{23}^{2} z_{45}}+\frac{\left\langle L_{233434} V_{1} V_{5} V_{6}\right\rangle}{z_{23} z_{34}^{2}} \\
& \left.+\frac{\left\langle L_{243434} V_{1} V_{5} V_{6}\right\rangle}{z_{24} z_{34}^{2}}+\frac{\left\langle L_{242434} V_{1} V_{5} V_{6}\right\rangle}{z_{24}^{2} z_{34}}+\frac{\left\langle L_{232334} V_{1} V_{5} V_{6}\right\rangle}{z_{23}^{2} z_{34}}\right\} .
\end{aligned}
$$

The last ten double-pole integrals and their kinematic factors will be considered separately below. Regarding the twenty four single-pole kinematic factors, fifteen can be obtained by $1 \leftrightarrow 5,2 \leftrightarrow 4$ relabellings of

$$
\begin{array}{lllll}
\left\langle L_{213141} V_{5} V_{6}\right\rangle, & \left\langle L_{213145} V_{6}\right\rangle, & \left\langle L_{213441} V_{5} V_{6}\right\rangle, & \left\langle L_{213445} V_{6}\right\rangle, & \left\langle L_{213541} V_{6}\right\rangle, \\
\left\langle L_{213545} V_{6}\right\rangle, & \left\langle L_{233141} V_{5} V_{6}\right\rangle, & \left\langle L_{233145} V_{6}\right\rangle, & \left\langle L_{233441} V_{5} V_{6}\right\rangle
\end{array}
$$

and it will now be shown that BCJ-like kinematic identities reduce the number of independent kinematics to only four.

\subsection{Single-pole integrands and BCJ-inspired technique}

The Bern-Carrasco-Johansson (BCJ) kinematic identities are relations among the kinematic factors associated to different kinematic poles in the field-theory scattering amplitudes [4]. In string theory, exploiting the independence of the CFT correlator on the order in which the OPE expansions are used one obtains BCJ-like relations for the kinematic factors associated to different hypergeometric integrals [25].

For example, one might start the CFT calculation using the OPE's of $U^{2}\left(z_{2}\right)$ to integrate out the conformal weight-one variables with $z_{2}$ dependence. Then the OPE's of $U^{3}\left(z_{3}\right)$ and $U^{4}\left(z_{4}\right)$ can be used in different order to get the $z_{3}$ and $z_{4}$ dependencies. The kinematic factors and integrands obtained with the two different orderings of OPE elimination are simply related by relabeling $3 \leftrightarrow 4$ in their analogous expressions for (3.2).

While relabeling the kinematic factors is straightforward, relabeling the $z_{i j}$ dependencies may introduce different single-pole integrals which are not part of the original set of twenty-four obtained with the first ordering. The end result of the CFT correlator being the same, there must be relations which allow them to be expressed in terms of the original integrands. ${ }^{6}$ In fact, the partial fraction identities listed in (B.5) provide such relations. For example, the integrand $I_{13} \equiv$ $1 /\left(z_{25} z_{34} z_{41}\right)$ is relabeled to $1 /\left(z_{25} z_{43} z_{31}\right) \equiv-I_{52}$, which is not in the original set. But using (B.5) it can be rewritten as

$$
I_{52}=I_{13}-I_{5}
$$

and both $I_{13}$ and $I_{5} \equiv 1 /\left(z_{25} z_{31} z_{41}\right)$ are present in (3.2). By considering an augmented set of 63 integrands in Appendix B, all new integrands obtained via relabeling in this BCJ-inspired technique can be rewritten in terms of linear combinations of the original twenty-four ${ }^{7}$ integrands

\footnotetext{
6 The relations must hold for the $\left(z_{i j} z_{k l} z_{m n}\right)^{-1}$ before doing the $z_{2}, z_{3}, z_{4}$ integration because they refer to the properties of the CFT correlator. In particular, the total derivative equations of Appendix B are not necessary to get BCJ-like relations for the kinematic factors.

7 This augmented set method can be used to find the $(N-3)$ ! basis of integrals for $N$-point amplitude computations [36].
} 
appearing in (3.2). Therefore, subtracting the amplitudes computed with different orderings for the OPE's gives relations among kinematic factors; where some are originally present in (3.2) while others are simple relabellings of those. It turns out that this method allows the more involved kinematic factors of (3.2) to be expressed in terms of simpler ones through the following BCJ-like kinematic identities

$$
\begin{aligned}
& L_{213441}=L_{214131}-L_{213141}, \quad L_{213445}=L_{214535}-L_{213545}, \\
& L_{233141}=L_{312141}-L_{213141}, \quad L_{233145}=L_{312145}-L_{213145}, \\
& L_{233441}=L_{213141}-L_{312141}+L_{413121}-L_{412131} .
\end{aligned}
$$

This method is particularly efficient in reducing the amount of work. For example, the explicit computation of $L_{233441}$ is rather tedious because it involves OPE's between three integrated vertices $U^{i}\left(z_{i}\right)$ among themselves, as opposed to the simpler cases such as $L_{213141}$ and $L_{213545}$ where $U^{i}\left(z_{i}\right)$ collides with $V^{j}\left(z_{j}\right)$.

Therefore the basis from which all 24 single-pole kinematic factors can be obtained by simple relabeling is given by,

$$
\begin{aligned}
& \left\langle L_{213141} V_{5} V_{6}\right\rangle, \\
& \left\langle L_{213145} V_{6}\right\rangle=\left\langle L_{54} L_{2131} V_{6}\right\rangle, \\
& \left\langle L_{213545} V_{6}\right\rangle=\left\langle L_{3545} L_{12} V_{6}\right\rangle, \\
& \left\langle L_{213541} V_{6}\right\rangle=\left\langle L_{53} L_{2141} V_{6}\right\rangle,
\end{aligned}
$$

where the equalities for the last three lines can be show by explicit computations.

\subsection{Double-pole integrands and total derivative techniques}

Similarly as in the five-point computation, using the SYM equations of motion the kinematic factors of double-pole integrals can be rewritten in such a way as to contain overall factors of $(1+$ $\left.s_{i j}\right)$. These are precisely the factors which cancel the tachyon poles $\left(1+s_{i j}\right)^{-1}$ in double-pole integrals and allow them to be rewritten as linear combinations of single-pole integrals using total derivative relations of Appendix B. However, the six-point amplitude additionally involves integrals with tachyon poles $\left(1+s_{i j k}\right)^{-1}$ in the $t_{i}$ variables which are slightly more difficult to cancel.

It will be convenient to separate the double-pole contributions in (3.2) in two distinct sets. Each of the last four terms of (3.2)

$$
\begin{aligned}
& \int_{0}^{1} d z_{2} \int_{z_{2}}^{1} d z_{3} \int_{z_{3}}^{1} d z_{4} \prod_{i<j}^{6} z_{i j}^{-s_{i j}}\left\{\frac{\left\langle L_{233434} V_{1} V_{5} V_{6}\right\rangle}{z_{23} z_{34}^{2}}+\frac{\left\langle L_{243434} V_{1} V_{5} V_{6}\right\rangle}{z_{24} z_{34}^{2}}\right. \\
& \left.+\frac{\left\langle L_{242434} V_{1} V_{5} V_{6}\right\rangle}{z_{24}^{2} z_{34}}+\frac{\left\langle L_{232334} V_{1} V_{5} V_{6}\right\rangle}{z_{23}^{2} z_{34}}\right\}
\end{aligned}
$$

for itself is proportional to the tachyon pole $\left(1+t_{2}\right)^{-1}$ due to the double-pole integrals $\mathcal{I}_{27}, \mathcal{I}_{28}, \mathcal{I}_{29}$ and $\mathcal{I}_{36}$. After some manipulations which are explained in Appendix B.3, the double-pole contributions of (3.7) become 


$$
\begin{aligned}
& \int_{0}^{1} d z_{2} \int_{z_{2}}^{1} d z_{3} \int_{z_{3}}^{1} d z_{4} \prod_{i<j}^{6} z_{i j}^{-s_{i j}}\left\{\frac{\left\langle L_{233434} V_{1} V_{5} V_{6}\right\rangle}{z_{23} z_{34}^{2}}+\frac{\left\langle L_{243434} V_{1} V_{5} V_{6}\right\rangle}{z_{24} z_{34}^{2}}\right. \\
&\left.\quad+\frac{\left\langle L_{242434} V_{1} V_{5} V_{6}\right\rangle}{z_{24}^{2} z_{34}}+\frac{\left\langle L_{232334} V_{1} V_{5} V_{6}\right\rangle}{z_{23}^{2} z_{34}}\right\} \\
&=\left\langle R_{243} V_{1} V_{5} V_{6}\right\rangle\left(-s_{12} \mathcal{I}_{32}-s_{12} \mathcal{I}_{16}+s_{12} \mathcal{I}_{10}-s_{25} \mathcal{I}_{33}-s_{25} \mathcal{I}_{20}+s_{25} \mathcal{I}_{14}\right) \\
& \quad+\left\langle R_{234} V_{1} V_{5} V_{6}\right\rangle\left(-s_{12} \mathcal{I}_{30}+s_{12} \mathcal{I}_{22}+s_{12} \mathcal{I}_{10}-s_{12} \mathcal{I}_{1}\right. \\
&\left.-s_{25} \mathcal{I}_{31}+s_{25} \mathcal{I}_{26}+s_{25} \mathcal{I}_{14}-s_{25} \mathcal{I}_{8}\right) \\
&+\left\langle\left[D_{34}\left(k^{3} \cdot A^{2}\right)+D_{23}\left(k^{2} \cdot A^{4}\right)-D_{24}\left(k^{2} \cdot A^{3}\right)\right] V_{1} V_{5} V_{6}\right\rangle\left(s_{14} \mathcal{I}_{30}+s_{45} \mathcal{I}_{31}\right) \\
&-\left\langle\left[D_{34}\left(k^{4} \cdot A^{2}\right)-D_{23}\left(k^{2} \cdot A^{4}\right)+D_{24}\left(k^{2} \cdot A^{3}\right)\right] V_{1} V_{5} V_{6}\right\rangle\left(s_{13} \mathcal{I}_{32}+s_{35} \mathcal{I}_{33}\right) .
\end{aligned}
$$

These are simply corrections to the kinematics of the single-pole integrals with $R_{i j k}$ and $O_{i j k}$ building blocks.

The other set of double-pole integral contribution from (3.2) is given by

$$
\begin{aligned}
& \int_{0}^{1} d z_{2} \int_{z_{2}}^{1} d z_{3} \int_{z_{3}}^{1} d z_{4} \prod_{i<j}^{6} z_{i j}^{-s_{i j}}\left\{\frac{\left\langle L_{213434} V_{5} V_{6}\right\rangle}{z_{21} z_{34}^{2}}+\frac{\left\langle L_{253434} V_{1} V_{6}\right\rangle}{z_{25} z_{34}^{2}}+\frac{\left\langle L_{242431} V_{5} V_{6}\right\rangle}{z_{24}^{2} z_{31}}\right. \\
& \left.+\frac{\left\langle L_{242435} V_{1} V_{6}\right\rangle}{z_{24}^{2} z_{35}}+\frac{\left\langle L_{232341} V_{5} V_{6}\right\rangle}{z_{23}^{2} z_{41}}+\frac{\left\langle L_{232345} V_{1} V_{6}\right\rangle}{z_{23}^{2} z_{45}}\right\} .
\end{aligned}
$$

By doing the explicit OPE computations with the conventions of Section 2 one arrives at

$$
\begin{array}{ll}
L_{213434}=\left(1+s_{34}\right) L_{12} D_{34}, & L_{253434}=-\left(1+s_{34}\right) L_{52} D_{34}, \\
L_{232341}=\left(1+s_{23}\right) L_{14} D_{23}, & L_{232345}=-\left(1+s_{23}\right) L_{54} D_{23}, \\
L_{242431}=\left(1+s_{24}\right) L_{13} D_{24}, & L_{242435}=-\left(1+s_{24}\right) L_{53} D_{24} .
\end{array}
$$

The factors of $\left(1+s_{i j}\right)$ in $(3.10)$ cancel all the tachyon poles and allow the application of total derivative relations from Appendix B to rewrite (3.9) using only single-pole integrals.

\subsection{The six-point amplitude with BRST building blocks}

As explained in the previous subsection, the relations from Appendix B between generalized Euler integrals allow to rewrite the ten double-pole integrals as corrections to the 24 single poles integrals. After going through a long computation one checks that these double-pole corrections are precisely of the form which replace the superfields $L_{i j}, L_{j i k i}$ and $L_{j i k i l i}$ by their corresponding BRST building blocks $T_{i j}, T_{i j k}$ and $T_{i j k l}$ defined in Section 2.2. Furthermore, the resulting integrals accompanying these BRST kinematic factors can be rewritten using the partial fraction solutions (B.5), e.g. $\left\langle T_{1234} V_{5} V_{6}\right\rangle\left(\mathcal{I}_{30}-\mathcal{I}_{22}-\mathcal{I}_{10}+\mathcal{I}_{1}\right)=\left\langle T_{1234} V_{5} V_{6}\right\rangle \mathcal{I}_{61}$, leading to a surprisingly simple answer,

$$
\begin{aligned}
& A(1,2,3,4,5,6) \\
&=\left\langle T_{1234} V_{5} V_{6}\right\rangle \mathcal{I}_{61}-\left\langle T_{1243} V_{5} V_{6}\right\rangle \mathcal{I}_{59}-\left\langle T_{1324} V_{5} V_{6}\right\rangle \mathcal{I}_{55}-\left\langle T_{1342} V_{5} V_{6}\right\rangle \mathcal{I}_{32} \\
&-\left\langle T_{1423} V_{5} V_{6}\right\rangle \mathcal{I}_{51}+\left\langle T_{1432} V_{5} V_{6}\right\rangle \mathcal{I}_{30}-\left\langle T_{5234} V_{1} V_{6}\right\rangle \mathcal{I}_{43}+\left\langle T_{5243} V_{1} V_{6}\right\rangle \mathcal{I}_{40} \\
&+\left\langle T_{5324} V_{1} V_{6}\right\rangle \mathcal{I}_{45}+\left\langle T_{5342} V_{1} V_{6}\right\rangle \mathcal{I}_{33}+\left\langle T_{5423} V_{1} V_{6}\right\rangle \mathcal{I}_{44}-\left\langle T_{5432} V_{1} V_{6}\right\rangle \mathcal{I}_{31}
\end{aligned}
$$




$$
\begin{aligned}
& -\left\langle T_{123} T_{45} V_{6}\right\rangle \mathcal{I}_{60}-\left\langle T_{124} T_{35} V_{6}\right\rangle \mathcal{I}_{58}+\left\langle T_{132} T_{45} V_{6}\right\rangle \mathcal{I}_{25}-\left\langle T_{134} T_{25} V_{6}\right\rangle \mathcal{I}_{52} \\
& +\left\langle T_{142} T_{35} V_{6}\right\rangle \mathcal{I}_{19}+\left\langle T_{143} T_{25} V_{6}\right\rangle \mathcal{I}_{13}+\left\langle T_{253} T_{14} V_{6}\right\rangle \mathcal{I}_{49}+\left\langle T_{254} T_{13} V_{6}\right\rangle \mathcal{I}_{53} \\
& -\left\langle T_{352} T_{14} V_{6}\right\rangle \mathcal{I}_{23}+\left\langle T_{354} T_{12} V_{6}\right\rangle \mathcal{I}_{57}-\left\langle T_{452} T_{13} V_{6}\right\rangle \mathcal{I}_{17}-\left\langle T_{453} T_{12} V_{6}\right\rangle \mathcal{I}_{11} .
\end{aligned}
$$

Writing $\left\langle T_{i j k l} V_{m} V_{n}\right\rangle=-\left\langle T_{i j k l} Q T_{m n}\right\rangle / s_{m n}$ and integrating the BRST charge by parts using (2.26), many terms cancel due to the total derivative relations obeyed by the integrals and one arrives at the expression (1.1) presented in Section 1,

$$
\begin{aligned}
A(1,2,3,4,5,6)= & \left\langle T_{12} T_{34} T_{56}\right\rangle F_{1}^{\prime}+\left\langle T_{54} T_{32} T_{16}\right\rangle F_{1} \\
& +\left\langle T_{14} T_{23} T_{56}\right\rangle F_{2}^{\prime}+\left\langle T_{52} T_{43} T_{16}\right\rangle F_{2}+\left\langle T_{13} T_{24} T_{56}\right\rangle F_{3}^{\prime}+\left\langle T_{53} T_{42} T_{16}\right\rangle F_{3} \\
& +\left\langle T_{123} E_{456}\right\rangle F_{4}+\left\langle T_{543} E_{216}\right\rangle F_{4}^{\prime}+\left\langle T_{324} E_{561}\right\rangle F_{5}+\left\langle T_{342} E_{561}\right\rangle F_{5}^{\prime} \\
& +\left\langle T_{435} E_{216}\right\rangle F_{6}+\left\langle T_{231} E_{456}\right\rangle F_{6}^{\prime}+\left\langle T_{325} E_{416}\right\rangle F_{7}+\left\langle T_{341} E_{256}\right\rangle F_{7}^{\prime} \\
& +\left\langle T_{124} E_{356}\right\rangle F_{8}+\left\langle T_{542} E_{316}\right\rangle F_{8}^{\prime}+\left\langle T_{352} E_{416}\right\rangle F_{9}+\left\langle T_{314} E_{256}\right\rangle F_{9}^{\prime} \\
& +\left\langle T_{241} E_{356}\right\rangle F_{10}+\left\langle T_{425} E_{316}\right\rangle F_{10}^{\prime},
\end{aligned}
$$

where the integrals $F_{i}$ and $F_{i}^{\prime}$ are defined by

$$
\begin{aligned}
& F_{1}=-\frac{s_{13} \mathcal{I}_{25}+s_{1} \mathcal{I}_{60}}{s_{6}}, \quad F_{1}^{\prime}=-\frac{s_{35} \mathcal{I}_{57}+s_{4} \mathcal{I}_{11}}{s_{5}}, \\
& F_{2}=-\frac{s_{14} \mathcal{I}_{13}+s_{13} \mathcal{I}_{52}}{s_{6}}, \quad F_{2}^{\prime}=-\frac{s_{25} \mathcal{I}_{49}+s_{35} \mathcal{I}_{23}}{s_{5}}, \\
& F_{3}=-\frac{s_{14} \mathcal{I}_{19}+s_{1} \mathcal{I}_{58}}{s_{6}}, \quad F_{3}^{\prime}=-\frac{s_{25} \mathcal{I}_{53}+s_{4} \mathcal{I}_{17}}{s_{5}}, \\
& F_{4}=s_{4} \mathcal{I}_{2}, \quad F_{4}^{\prime}=-s_{1} \mathcal{I}_{4}, \quad F_{6}=s_{1} \mathcal{I}_{57}, \quad F_{6}^{\prime}=s_{4} \mathcal{I}_{25}, \\
& F_{7}=s_{14} \mathcal{I}_{49}, \quad F_{7}^{\prime}=s_{25} \mathcal{I}_{13}, \quad F_{8}=s_{35} \mathcal{I}_{3}, \quad F_{8}^{\prime}=-s_{13} \mathcal{I}_{6}, \\
& F_{9}=s_{14} \mathcal{I}_{7}, \quad F_{9}^{\prime}=-s_{25} \mathcal{I}_{5}, \quad F_{10}=s_{35} \mathcal{I}_{19}, \quad F_{10}^{\prime}=s_{13} \mathcal{I}_{53}, \\
& F_{5}=s_{1} \mathcal{I}_{62}+s_{13} \mathcal{I}_{55}+s_{14} \mathcal{I}_{51}, \quad F_{5}^{\prime}=s_{1} \mathcal{I}_{59}+s_{13} \mathcal{I}_{32}+s_{14} \mathcal{I}_{48} .
\end{aligned}
$$

The explicit $\alpha^{\prime}$-expansions of the integrals $F_{i}$ obtained using the methods explained in [21] are written down in Appendix B.

\subsection{Field theory limit}

Plugging in the momenta expansions for the $F_{i}, F_{i}^{\prime}$ integrals appearing in (1.1) and taking their limit as $\alpha^{\prime} \rightarrow 0$ gives the field-theory super-Yang-Mills six-point tree amplitude

$$
\begin{aligned}
& A_{\mathrm{SYM}}(1,2,3,4,5,6)=\frac{\left\langle T_{12} T_{34} T_{56}\right\rangle}{s_{1} s_{3} s_{5}}+\frac{\left\langle T_{54} T_{32} T_{16}\right\rangle}{s_{2} s_{4} s_{6}} \\
& \quad+\frac{\left\langle T_{123} E_{456}\right\rangle}{s_{1} t_{1}}+\frac{\left\langle T_{543} E_{216}\right\rangle}{s_{4} t_{3}}+\frac{\left\langle T_{321} E_{456}\right\rangle}{s_{2} t_{1}}+\frac{\left\langle T_{345} E_{216}\right\rangle}{s_{3} t_{3}}+\frac{\left\langle T_{432} E_{561}\right\rangle}{s_{3} t_{2}}+\frac{\left\langle T_{234} E_{561}\right\rangle}{s_{2} t_{2}},
\end{aligned}
$$

which agrees with the Ansatz proposed in [27], and therefore proves it. To see this first rewrite the six-point expression of [27] using the $E_{i j k}$ building blocks to obtain 


$$
\begin{aligned}
& A_{\mathrm{SYM}}^{\text {ansatz }}(1,2,3,4,5,6)=\frac{\left\langle T_{12} T_{34} T_{56}\right\rangle}{s_{1} s_{3} s_{5}}+\frac{\left\langle T_{54} T_{32} T_{16}\right\rangle}{s_{2} s_{4} s_{6}} \\
& \quad+\frac{1}{2}\left(\frac{T_{123} E_{456}}{s_{1} t_{1}}+\frac{T_{234} E_{561}}{s_{2} t_{2}}+\frac{T_{345} E_{612}}{s_{3} t_{3}}+\frac{T_{456} E_{123}}{s_{4} t_{1}}+\frac{T_{561} E_{234}}{s_{5} t_{2}}+\frac{T_{612} E_{345}}{s_{6} t_{3}}\right) \\
& \quad-\frac{1}{2}\left(\frac{T_{126} E_{345}}{s_{1} t_{3}}+\frac{T_{231} E_{456}}{s_{2} t_{1}}+\frac{T_{342} E_{561}}{s_{3} t_{2}}+\frac{T_{453} E_{612}}{s_{4} t_{3}}+\frac{T_{564} E_{123}}{s_{5} t_{1}}+\frac{T_{615} E_{234}}{s_{6} t_{2}}\right) .
\end{aligned}
$$

To relate the last two lines of (3.15) with the last line of (3.14) it is sufficient to consider the identities

$$
0=\left\langle Q\left[\left(\frac{T_{123}}{s_{1}}+\frac{T_{321}}{s_{2}}\right)\left(\frac{T_{456}}{s_{4}}+\frac{T_{654}}{s_{5}}\right)\right]\right\rangle, \quad Q\left[\frac{T_{123}}{s_{12}}+\frac{T_{321}}{s_{23}}\right]=-s_{123} E_{123},
$$

and relabellings of it. Using (3.16) and the momentum conservation relation $s_{123}=s_{456}$ one obtains, for example

$$
\left\langle\left(\frac{T_{456}}{s_{4}}-\frac{T_{564}}{s_{5}}\right) E_{123}\right\rangle=\left\langle\left(\frac{T_{123}}{s_{1}}-\frac{T_{231}}{s_{2}}\right) E_{456}\right\rangle
$$

which together with its relabellings allow one to prove that (3.15) and (3.14) are equal; $A_{\mathrm{SYM}}^{\text {ansatz }}=$ $A_{\mathrm{SYM}}$. Each term of the field-theory limit expression (3.14) can be associated to Feynman diagrams which use only three-point vertices as in the arguments of [4]. The explicit mapping is shown in Fig. 4 of the Appendix D.

\section{BCJ identities in superspace}

In field theory the kinematic factors of an $N$-point tree-level gluon amplitude can be rearranged such that the form of any partial amplitude becomes rather simple. More precisely, kinematic factors corresponding to diagrams with purely cubic interactions can be chosen and any subamplitude is organized as a sum over terms describing these diagrams [4]. The latter are specified by some numerator factors and their corresponding propagator structure. The total number of such numerator factors or channels is given by $(2 N-5)$ !!, while the number of independent factors is $(N-2)$ ! [4]. Any contact term may be absorbed into the numerator factors of the diagrams. Moreover, it has been argued in [4], that kinematic numerator identities impose additional constraints. As a consequence in field-theory the number of independent color-ordered $N$-point amplitudes at tree-level is $(N-3)$ ! [4]. As demonstrated in [5,6] this result may be easily anticipated from string theory.

\subsection{Extended BCJ relations from monodromy relations}

After imposing cyclic symmetry, reflection and parity symmetries there are $\frac{1}{2}(N-1)$ ! different color ordered subamplitudes. Furthermore, after applying Kleiss-Kuijf relations we end up at $(N-2)$ ! independent subamplitudes. As a basis we may choose the following $(N-2)$ ! elements $A\left(1,2_{\sigma}, \ldots,(N-1)_{\sigma}, N\right)$ with the permutation $\sigma \in S_{N-2}[37,38]$. Hence, for the case of interest with $N=6$ we need to specify 24 subamplitudes and parametrize the latter according to their pole structure, which arises from diagrams with only three-vertices contributing. To each amplitude 14 terms or diagrams contribute. The topological structure of the latter is depicted in the following two figures. For the first amplitude we make the Ansatz 


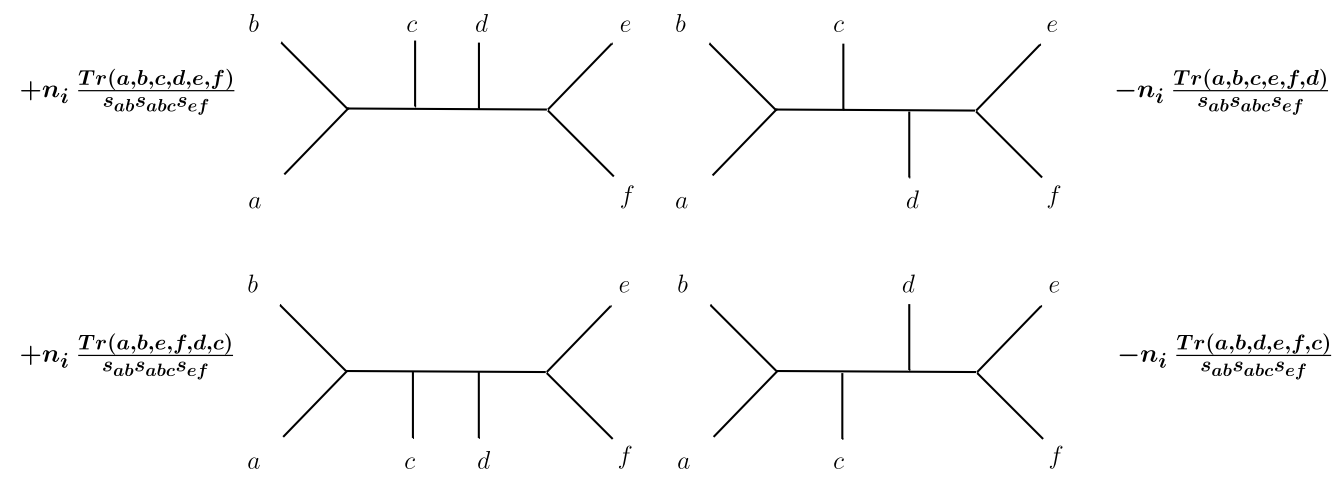

Fig. 2. Diagrams giving rise to the numerator factor $n_{i}$.

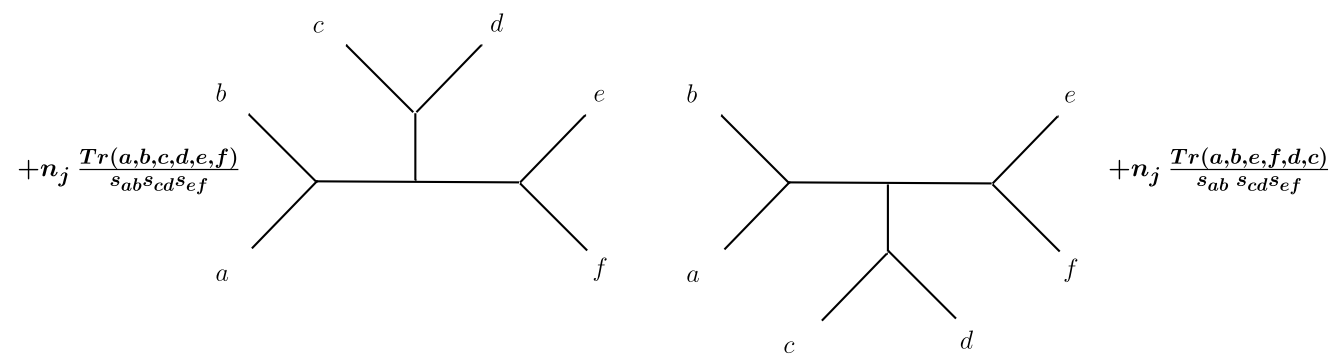

Fig. 3. Diagrams giving rise to the numerator factor $n_{j}$.

$$
\begin{aligned}
A(1,2,3,4,5,6)= & \frac{n_{1}(2345)}{s_{12} s_{34} s_{56}}+\frac{n_{2}(2345)}{s_{23} s_{45} s_{123}}+\frac{n_{3}(2345)}{s_{12} s_{45} s_{123}}+\frac{n_{4}(2345)}{s_{12} s_{56} s_{123}}+\frac{n_{5}(2345)}{s_{23} s_{56} s_{123}} \\
& +\frac{n_{6}(2345)}{s_{23} s_{56} s_{156}}+\frac{n_{7}(2345)}{s_{34} s_{56} s_{156}}+\frac{n_{1}^{\prime}(2345)}{s_{16} s_{23} s_{45}}+\frac{n_{2}^{\prime}(2345)}{s_{12} s_{34} s_{126}}+\frac{n_{3}^{\prime}(2345)}{s_{12} s_{45} s_{126}} \\
& +\frac{n_{4}^{\prime}(2345)}{s_{16} s_{45} s_{126}}+\frac{n_{5}^{\prime}(2345)}{s_{16} s_{34} s_{126}}+\frac{n_{6}^{\prime}(2345)}{s_{16} s_{34} s_{156}}+\frac{n_{7}^{\prime}(2345)}{s_{16} s_{23} s_{156}}
\end{aligned}
$$

with the numerator factors $n_{i}$. In the remaining 23 subamplitudes we have to take into account, that a numerator factor $n_{i}$ may contribute to various other subamplitudes. In Figs. 2 and 3 we display diagrams, which give rise to the same numerator factors $n_{i}$ and $n_{j}$, respectively. Taking this fact into account yields:

$$
\begin{aligned}
A(1,2,3,5,4,6)= & \frac{n_{1}(2354)}{s_{12} s_{35} s_{46}}-\frac{n_{2}(2345)}{s_{23} s_{45} s_{123}}-\frac{n_{3}(2345)}{s_{12} s_{45} s_{123}}+\frac{n_{4}(2354)}{s_{12} s_{46} s_{123}}+\frac{n_{5}(2354)}{s_{23} s_{46} s_{123}} \\
& +\frac{n_{6}(2354)}{s_{23} s_{46} s_{146}}+\frac{n_{7}(2354)}{s_{35} s_{46} s_{146}}-\frac{n_{1}^{\prime}(2345)}{s_{16} s_{23} s_{45}}+\frac{n_{2}^{\prime}(2354)}{s_{12} s_{35} s_{126}}-\frac{n_{3}^{\prime}(2345)}{s_{12} s_{45} s_{126}} \\
& -\frac{n_{4}^{\prime}(2345)}{s_{16} s_{45} s_{126}}+\frac{n_{5}^{\prime}(2354)}{s_{16} s_{35} s_{126}}+\frac{n_{6}^{\prime}(2354)}{s_{16} s_{35} s_{146}}+\frac{n_{7}^{\prime}(2354)}{s_{16} s_{23} s_{146}}, \\
& \vdots \\
A(1,5,4,3,2,6)= & -\frac{n_{1}(5342)}{s_{15} s_{26} s_{34}}+\frac{n_{2}(4523)}{s_{23} s_{45} s_{145}}-\frac{n_{3}(5423)}{s_{15} s_{23} s_{145}}+\frac{n_{4}(5432)}{s_{15} s_{26} s_{145}}-\frac{n_{5}(4532)}{s_{26} s_{45} s_{145}}
\end{aligned}
$$




$$
\begin{aligned}
& +\frac{n_{6}(3452)}{s_{26} s_{34} s_{126}}+\frac{n_{7}(3452)}{s_{26} s_{45} s_{126}}-\frac{n_{1}^{\prime}(2345)}{s_{16} s_{23} s_{45}}+\frac{n_{2}^{\prime}(5234)}{s_{15} s_{23} s_{156}}+\frac{n_{3}^{\prime}(5234)}{s_{15} s_{34} s_{156}} \\
& -\frac{n_{4}^{\prime}(2345)}{s_{16} s_{45} s_{126}}-\frac{n_{5}^{\prime}(2345)}{s_{16} s_{34} s_{126}}-\frac{n_{6}^{\prime}(2345)}{s_{16} s_{34} s_{156}}-\frac{n_{7}^{\prime}(2345)}{s_{16} s_{23} s_{156}}
\end{aligned}
$$

The parametrization of the remaining 21 subamplitudes is given in Appendix C. In total we need $7 ! !=105$ numerator factors $n_{i}$ to parametrize the 24 subamplitudes.

Now we shall make use of the monodromy relations, which give rise to non-trivial relations between various different subamplitudes [5,6]. One of these relations reads [6]:

$$
\begin{aligned}
& A(1,2,3,4,5,6)+e^{i \pi s_{12}} A(2,1,3,4,5,6)+e^{i \pi\left(s_{12}+s_{13}\right)} A(2,3,1,4,5,6) \\
& \quad+e^{i \pi\left(s_{12}+s_{13}+s_{14}\right)} A(2,3,4,1,5,6)+e^{i \pi\left(s_{12}+s_{13}+s_{14}+s_{15}\right)} A(2,3,4,5,1,6)=0 .
\end{aligned}
$$

A complete set can be obtained by permuting all open string labels. In the field-theory limit the real part of all these relations gives rise to the Kleiss-Kuijf relations. Hence, these relations allow to determine all 60 partial subamplitudes from the set of 24 given in (4.1) and (4.2). On the other hand, it has been argued in $[39,40]$, that the imaginary part of all relations gives rise to a set of equations, the so-called extended BCJ relations, which relate the numerator factors. In these equations three numerator factors $n_{i}$ constitute the triplets $X_{j}$. In the following we define the hundred triplets:

$$
\begin{aligned}
& X_{1}=n_{1}(2345)-n_{4}(2345)+n_{4}(2435), \quad X_{2}=n_{2}(2345)-n_{5}(2345)+n_{5}(2354), \\
& \vdots \\
& X_{99}=n_{4}(4532)-n_{4}(5432)-n_{5}(4532), \quad X_{100}=-n_{1}(5342)+n_{5}(3452)-n_{6}(3452) .
\end{aligned}
$$

The remaining 96 triplets can be found in Appendix C. From the imaginary part of the monodromy relations an independent set of $3 ! \cdot 3=18$ equations each containing 15 triplets (4.4) can be derived:

$$
\begin{gathered}
\frac{X_{1}}{s_{12} s_{56}}-\frac{X_{2}}{s_{23} s_{123}}-\frac{X_{3}}{s_{12} s_{123}}-\frac{X_{4}}{s_{23} s_{56}}+\frac{X_{5}}{s_{56} s_{156}}-\frac{X_{6}}{s_{23} s_{61}}+\frac{X_{7}}{s_{12} s_{126}}-\frac{X_{8}}{s_{61} s_{126}} \\
+\frac{X_{9}}{s_{61} s_{156}}+\frac{X_{10}}{s_{12} s_{35}}+\frac{X_{11}}{s_{35} s_{61}}+\frac{X_{12}}{s_{35} s_{124}}+\frac{X_{13}}{s_{56} s_{124}}+\frac{X_{14}}{s_{35} s_{146}}-\frac{X_{15}}{s_{23} s_{146}}=0 \\
\quad \vdots \\
\frac{X_{4}}{s_{23} s_{56}}+\frac{X_{20}}{s_{23} s_{145}}-\frac{X_{26}}{s_{45} s_{123}}-\frac{X_{27}}{s_{56} s_{123}}-\frac{X_{44}}{s_{45} s_{126}}-\frac{X_{45}}{s_{34} s_{126}}+\frac{X_{52}}{s_{34} s_{56}}-\frac{X_{64}}{s_{23} s_{45}} \\
-\frac{X_{70}}{s_{26} s_{45}}+\frac{X_{89}}{s_{26} s_{134}}-\frac{X_{82}}{s_{56} s_{134}}+\frac{X_{93}}{s_{23} s_{156}}+\frac{X_{98}}{s_{34} s_{156}}+\frac{X_{99}}{s_{26} s_{145}}-\frac{X_{100}}{s_{26} s_{34}}=0 .
\end{gathered}
$$

The remaining 16 equations are listed in Appendix C. In relations (4.5) each triplet $X_{i}$ is the numerator of a product of $N-4=2$ poles $s_{I}, s_{J}$. Eqs. (4.5) imply that the residue of each pole term must vanish, i.e.:

$$
\left.X_{i}\right|_{\text {residue }}=0 .
$$

However, the regular part of the triplets $X_{i}$, which is proportional to $s_{I} s_{J}$, may be non-vanishing. The choice $X_{i}=0$ would also be a solution of Eqs. (4.5), but it corresponds to a specific gauge 
choice. Eqs. (4.6) correspond to the set of color identities [4]. In fact, these identities give rise to 81 independent kinematic equations relating the 105 numerators $n_{i}$. Hence, in total there are 24 independent numerators $n_{i}$. On the other hand, the set of 18 extended BCJ relations (4.5) describes the general constraint on the numerator factors.

As will be demonstrated below, using the field-theory parametrization following from (3.11) together with the hook properties of the pure spinor building blocks $T_{i j k}$ and $T_{i j k l}$ it is possible to easily identify the explicit $(N-2)$ ! basis numerators. The explicit form of the supersymmetric numerator factors $n_{i}$ in (4.1) can be read off by comparing it with (3.14).

\subsection{Basis numerators for $N=5$}

The tree-level amplitude prescription (3.1) from string theory naturally suggests using the $(N-3)$ ! subamplitudes generated by the different orderings of the integrated vertices as a basis, i.e. $A\left(1,2_{\sigma}, \ldots,(N-2)_{\sigma}, N-1, N\right)$ with $\sigma \in S_{N-3}$ and positions $(1, N-1, N)$ fixed. For example, using the field-theory limit of the five-point amplitudes computed with the pure spinor formalism in [27,25], the $(N-3) !=2$ basis amplitudes can be written in terms of ten kinematic factors as ${ }^{8}$ :

$$
\begin{aligned}
& A_{\text {SYM }}(1,2,3,4,5) \\
& =\frac{\left\langle T_{123} V^{4} V^{5}\right\rangle}{s_{12} s_{45}}-\frac{\left\langle T_{234} V^{1} V^{5}\right\rangle}{s_{23} s_{51}}+\frac{\left\langle L_{12} L_{34} V^{5}\right\rangle}{s_{12} s_{34}}+\frac{\left\langle T_{321} V^{4} V^{5}\right\rangle}{s_{23} s_{45}}-\frac{\left\langle T_{432} V^{1} V^{5}\right\rangle}{s_{34} s_{51}}, \\
& A_{\text {SYM }}(1,3,2,4,5) \\
& =\frac{\left\langle T_{132} V^{4} V^{5}\right\rangle}{s_{13} s_{45}}-\frac{\left\langle T_{324} V^{1} V^{5}\right\rangle}{s_{23} s_{51}}+\frac{\left\langle L_{13} L_{24} V^{5}\right\rangle}{s_{13} s_{24}}+\frac{\left\langle T_{231} V^{4} V^{5}\right\rangle}{s_{23} s_{45}}-\frac{\left\langle T_{423} V^{1} V^{5}\right\rangle}{s_{24} s_{51}} .
\end{aligned}
$$

However, the number of independent kinematic factors is $(N-2) !=6$ because of the hook symmetries (2.22) of $T_{i j k}$,

$$
\begin{aligned}
& \left\langle T_{324} V^{1} V^{5}\right\rangle=-\left\langle T_{234} V^{1} V^{5}\right\rangle, \quad\left\langle T_{231} V^{4} V^{5}\right\rangle=-\left\langle T_{321} V^{4} V^{5}\right\rangle, \\
& \left\langle T_{132} V^{4} V^{5}\right\rangle=\left\langle T_{123} V^{4} V^{5}\right\rangle-\left\langle T_{321} V^{4} V^{5}\right\rangle, \\
& \left\langle T_{423} V^{1} V^{5}\right\rangle=\left\langle T_{432} V^{1} V^{5}\right\rangle-\left\langle T_{234} V^{1} V^{5}\right\rangle .
\end{aligned}
$$

\subsection{Basis numerators for $N=6$}

The six-point subamplitude in the field-theory limit (3.15) is expanded in terms of 14 poles, so the full $(N-3) !=6$ basis amplitudes would naively correspond to 84 kinematic factors. However, the pure spinor BRST building block form of the kinematic factors allows one to easily find the basis with $(N-2) !=24$ elements, in accord with the monodromy analysis of Section 4.1.

To see this it is convenient to use the field-theory limit of (3.11),

$$
\begin{aligned}
& A_{\mathrm{SYM}}(1,2,3,4,5,6) \\
& =\frac{\left\langle\left(T_{1234}-T_{1243}\right) V_{5} V_{6}\right\rangle}{s_{1} s_{3} s_{5}}+\frac{\left\langle\left(T_{5432}-T_{5423}\right) V_{1} V_{6}\right\rangle}{s_{2} s_{4} s_{6}}
\end{aligned}
$$

\footnotetext{
8 With the notation of [40] one can show that this parametrization leads to the vanishing of four triplets: $X_{3}=X_{5}=$ $X_{7}=n_{14}-n_{12}+n_{13}=0$ and the extended BCJ identities are satisfied.
} 


$$
\begin{aligned}
& +\frac{\left\langle\left(T_{132}-T_{123}\right) T_{45} V_{6}\right\rangle}{s_{2} s_{4} t_{1}}+\frac{\left\langle\left(T_{534}-T_{543}\right) T_{21} V_{6}\right\rangle}{s_{1} s_{3} t_{3}}-\frac{\left\langle T_{123} L_{45} V_{6}\right\rangle}{s_{1} s_{4} t_{1}}-\frac{\left\langle T_{453} L_{12} V_{6}\right\rangle}{s_{1} s_{4} t_{3}} \\
& +\frac{\left\langle T_{1234} V_{5} V_{6}\right\rangle}{s_{1} s_{5} t_{1}}+\frac{\left\langle T_{5432} V_{1} V_{6}\right\rangle}{s_{4} s_{6} t_{3}}+\frac{\left\langle\left(T_{1234}-T_{1324}\right) V_{5} V_{6}\right\rangle}{s_{2} s_{5} t_{1}}+\frac{\left\langle\left(T_{5432}-T_{5342}\right) V_{1} V_{6}\right\rangle}{s_{3} s_{6} t_{3}} \\
& +\frac{\left\langle\left(T_{1234}-T_{1324}-T_{1423}+T_{1432}\right) V_{5} V_{6}\right\rangle}{s_{2} s_{5} t_{2}} \\
& +\frac{\left\langle\left(T_{5432}-T_{5342}-T_{5243}+T_{5234}\right) V_{1} V_{6}\right\rangle}{s_{3} s_{6} t_{2}} \\
& +\frac{\left\langle\left(T_{1234}-T_{1243}-T_{1342}+T_{1432}\right) V_{5} V_{6}\right\rangle}{s_{3} s_{5} t_{2}} \\
& +\frac{\left\langle\left(T_{5432}-T_{5423}-T_{5324}+T_{5234}\right) V_{1} V_{6}\right\rangle}{s_{2} s_{6} t_{2}}
\end{aligned}
$$

The field-theory amplitude (4.9) is more conveniently written as

$$
\begin{aligned}
A(1,2,3,4,5,6)= & \frac{n_{1}(234)}{s_{1} s_{3} s_{5}}+\frac{n_{1}^{\prime}(234)}{s_{2} s_{4} s_{6}}+\frac{n_{2}(234)}{s_{2} s_{4} t_{1}}+\frac{n_{2}^{\prime}(234)}{s_{1} s_{3} t_{3}}+\frac{n_{3}(234)}{s_{1} s_{4} t_{1}}+\frac{n_{3}^{\prime}(234)}{s_{1} s_{4} t_{3}} \\
& +\frac{n_{4}(234)}{s_{1} s_{5} t_{1}}+\frac{n_{4}^{\prime}(234)}{s_{4} s_{6} t_{3}}+\frac{n_{5}(234)}{s_{2} s_{5} t_{1}}+\frac{n_{5}^{\prime}(234)}{s_{3} s_{6} t_{3}}+\frac{n_{6}(234)}{s_{2} s_{5} t_{2}}+\frac{n_{6}^{\prime}(234)}{s_{3} s_{6} t_{2}} \\
& +\frac{n_{7}(234)}{s_{3} s_{5} t_{2}}+\frac{n_{7}^{\prime}(234)}{s_{2} s_{6} t_{2}},
\end{aligned}
$$

where $n_{i}(j k l) \equiv n_{i}(j k l 5)$ such that the labels $(j k l)$ denote the ordering of the integrated vertices in the $(N-3) !=6$ basis of partial amplitudes. Their explicit form in terms of pure spinor BRST building blocks read

$$
\begin{aligned}
& n_{1}(234)=\left\langle\left(T_{1234}-T_{1243}\right) V_{5} V_{6}\right\rangle, \quad n_{2}(234)=\left\langle\left(T_{132}-T_{123}\right) T_{45} V_{6}\right\rangle, \\
& n_{3}(234)=-\left\langle T_{123} T_{45} V_{6}\right\rangle, \quad n_{4}(234)=\left\langle T_{1234} V_{5} V_{6}\right\rangle, \\
& n_{5}(234)=\left\langle\left(T_{1234}-T_{1324}\right) V_{5} V_{6}\right\rangle, \\
& n_{6}(234)=\left\langle\left(T_{1234}-T_{1324}-T_{1423}+T_{1432}\right) V_{5} V_{6}\right\rangle, \\
& n_{7}(234)=\left\langle\left(T_{1234}-T_{1243}-T_{1342}+T_{1432}\right) V_{5} V_{6}\right\rangle,
\end{aligned}
$$

while $n_{i}^{\prime}(i j k)$ is obtained from $n_{i}(i j k)$ by the parity transformation $1 \leftrightarrow 5,2 \leftrightarrow 4$.

It is now straightforward to check that the set of $12 \oplus 12^{\prime}$ kinematic factors

$$
\left\{n_{3}(i j k), n_{4}(i j k), n_{3}^{\prime}(i j k), n_{4}^{\prime}(i j k) /(i j k) \in \operatorname{perm}(234)\right\}
$$

is a basis from which all 84 kinematic numerators can be obtained. In fact, the explicit BCJ-like solution reads

$$
\begin{aligned}
& n_{1}(234)=n_{4}(234)-n_{4}(243), \quad n_{2}(234)=n_{3}(234)-n_{3}(243), \\
& n_{5}(234)=n_{4}(234)-n_{4}(324), \quad n_{6}(234)=n_{4}(234)-n_{4}(324)-n_{4}(423)+n_{4}(432), \\
& n_{7}(234)=n_{4}(234)-n_{4}(243)-n_{4}(342)+n_{4}(432) .
\end{aligned}
$$

Together with the permutations of (234) (with corresponding equations for $n_{i}^{\prime}(i j k)$ ), the solution (4.13) generates $30 \oplus 30^{\prime}$ equations which allow reducing the 84 kinematic factors down to 24 . One can also show that using the parametrization given by (4.9) together with the hook properties of $T_{i j k}$ and $T_{i j k l}, 59$ triplets defined in (4.4) trivially vanish 


$$
\begin{gathered}
X_{i}=0, \quad i=1,4-9,11-13,16-18,20,21,24,26-28,30,31,35-39,42,52-56,62, \\
\quad 63,70-75,77,78,80-82,84-92,95-97,99,100 .
\end{gathered}
$$

By applying [30] the 18 extended BCJ's (4.5) have explicitly been checked to be satisfied using the pure spinor representation (4.11) for the amplitudes in (4.2). In Appendix C it is explicitly shown that using the symmetry properties of $T_{i j k}$ and $T_{i j k l}$, the solution to $X_{i}=0$ for $i=1, \ldots, 100$ implies that all 105 numerators $n_{j}$ in the Kleiss-Kuijf basis can be expressed in terms of the basis (4.12).

\section{Acknowledgements}

We are grateful to Giuseppe Policastro for useful discussions. C.M. would like to thank the Werner-Heisenberg-Institut in München for hospitality and the friendly atmosphere during preparation of this work. C.M. and D.T. also thank the organizers of the Amsterdam String Theory Workshop, where some discussions took place in an inspiring location. C.M. thanks the partial financial support from the MPG and acknowledges support by the Deutsch-Israelische Projektkooperation (DIP H52). St.St. would like to thank the Albert-Einstein-Institut in Potsdam and in particular Hermann Nicolai and Stefan Theisen for invitation and partial support during completion of this work. The diagrams have been created by the program JaxoDraw [41].

\section{Appendix A. The superfields $L_{21}, L_{2131}$ and $L_{213141}$}

Using the OPE's of Section 2 and a few BRST manipulations together with the SYM equations of motion it follows that ${ }^{9}$

$$
\lim _{z_{2} \rightarrow z_{1}} V^{1}\left(z_{1}\right) U^{2}\left(z_{2}\right) \rightarrow \frac{L_{12}}{z_{12}}, \quad \lim _{z_{2}, z_{3} \rightarrow z_{1}} V^{1}\left(z_{1}\right) U^{2}\left(z_{2}\right) U^{3}\left(z_{3}\right) \rightarrow \frac{L_{2131}}{z_{21} z_{31}},
$$

where $[15,25]$

$$
\begin{aligned}
& L_{12}=A_{m}^{1}\left(\lambda \gamma^{m} W^{2}\right)+V^{1}\left(k^{1} \cdot A^{2}\right), \\
& L_{2131}=L_{12}\left(\left(k^{1}+k^{2}\right) \cdot A^{3}\right)+\left(\lambda \gamma^{m} W^{3}\right)\left[A_{m}^{1}\left(k^{1} \cdot A^{2}\right)+A^{1 n} \mathcal{F}_{m n}^{2}-\left(W^{1} \gamma_{m} W^{2}\right)\right] .
\end{aligned}
$$

Finally, a long computation leads to

$$
\lim _{z_{2}, z_{3}, z_{4} \rightarrow z_{1}} V^{1}\left(z_{1}\right) U^{2}\left(z_{2}\right) U^{3}\left(z_{3}\right) U^{4}\left(z_{4}\right) \rightarrow \frac{L_{213141}}{z_{21} z_{31} z_{41}}
$$

where

$$
\begin{aligned}
L_{213141}= & -L_{2131}\left[A_{4} \cdot\left(k_{1}+k_{2}+k_{3}\right)\right]+\left(A_{3} \cdot\left(k_{1}+k_{2}\right)\right) \\
& \times\left[\left(A_{1} \cdot k_{2}\right) A_{m}^{2}-\left(A_{1} \cdot A_{2}\right) k_{m}^{2}-A_{m}^{1}\left(A_{2} \cdot k_{1}\right)+\left(W_{1} \gamma_{m} W_{2}\right)\right]\left(\lambda \gamma^{m} W_{4}\right) \\
& +\left(\lambda \gamma^{m} W_{4}\right)\left[\left(A_{1} \cdot k_{2}\right)\left(A_{2} \cdot A_{3}\right) k_{m}^{3}-\left(A_{1} \cdot k_{2}\right)\left(W_{2} \gamma^{m} W_{3}\right)\right. \\
& -\left(A_{1} \cdot A_{2}\right)\left(k_{2} \cdot A_{3}\right) k_{m}^{3}+\left(A_{1} \cdot A_{2}\right)\left(k_{2} \cdot k_{3}\right) A_{m}^{3}-\left(A_{1} \cdot k_{2}\right)\left(A_{2} \cdot k_{3}\right) A_{m}^{3}
\end{aligned}
$$

\footnotetext{
9 It was pointed out in $[15,25]$ that extracting $L_{i j}$ and $L_{j i k i}$ from the $V^{i} U^{j}$ and $V^{i} U^{j} U^{k}$ OPE's additionally involve $Q$ integration by parts. The same happens for $L_{j i k i l i}$, but the arising extra terms of schematic form $s_{i j}\left(A^{k} W^{l}\right)$, $s_{i j}\left(A^{k} \gamma^{p q} W^{l}\right)$ and $s_{i j}\left(W^{k} A_{l} W^{m}\right)$ turn out to cancel in the overall $6 \mathrm{pt}$ amplitude.
} 


$$
\begin{aligned}
& +\left(A_{1} \cdot k_{3}\right)\left(k_{1} \cdot A_{2}\right) A_{m}^{3}-\left(A_{1} \cdot A_{3}\right)\left(k_{1} \cdot A_{2}\right) k_{m}^{3}+\left(W_{1} \gamma^{n} W_{2}\right) \mathcal{F}_{m n}^{3} \\
& \left.+\frac{1}{4}\left(W_{2} \gamma_{p q} \gamma_{m} W_{3}\right) \mathcal{F}_{1}^{p q}-\frac{1}{4}\left(W_{1} \gamma_{p q} \gamma_{m} W_{3}\right) \mathcal{F}_{2}^{p q}+\left(W_{1} \gamma_{m} W_{3}\right)\left(k_{1} \cdot A_{2}\right)\right] .
\end{aligned}
$$

\section{Appendix B. Six-point integrals}

A direct computation of the six-point amplitude with the pure spinor formalism requires 34 triple integrals of the form

$$
\mathcal{I}_{k}=\int d z_{2} \int d z_{3} \int d z_{4} \prod_{i<j}^{6} z_{i j}^{-s_{i j}} I_{k},
$$

where $I_{k}, k=1, \ldots, 34$ will be written below. However, it is convenient to consider an augmented set of 63 integrals by including $I_{k}, k=35, \ldots, 63$ which allow the definition of a system of equations which can be used to simplify the amplitude considerably. This convenient set of $\left\{I_{k}\right\}$ is given by

$$
\begin{aligned}
& I_{1}=\frac{1}{z_{21} z_{31} z_{41}}, \quad I_{2}=\frac{1}{z_{21} z_{31} z_{45}}, \quad I_{3}=\frac{1}{z_{21} z_{35} z_{41}}, \quad I_{4}=\frac{1}{z_{21} z_{35} z_{45}}, \\
& I_{5}=\frac{1}{z_{25} z_{31} z_{41}}, \quad I_{6}=\frac{1}{z_{25} z_{31} z_{45}}, \quad I_{7}=\frac{1}{z_{25} z_{35} z_{41}}, \quad I_{8}=\frac{1}{z_{25} z_{35} z_{45}}, \\
& I_{9}=\frac{1}{z_{21} z_{34}^{2}}, \quad I_{10}=\frac{1}{z_{21} z_{34} z_{41}}, \quad I_{11}=\frac{1}{z_{21} z_{34} z_{45}}, \quad I_{12}=\frac{1}{z_{25} z_{34}^{2}}, \\
& I_{13}=\frac{1}{z_{25} z_{34} z_{41}}, \quad I_{14}=\frac{1}{z_{25} z_{34} z_{45}}, \quad I_{15}=\frac{1}{z_{31} z_{24}^{2}}, \quad I_{16}=\frac{1}{z_{31} z_{24} z_{41}}, \\
& I_{17}=\frac{1}{z_{31} z_{24} z_{45}}, \quad I_{18}=\frac{1}{z_{35} z_{24}^{2}}, \quad I_{19}=\frac{1}{z_{35} z_{24} z_{41}}, \quad I_{20}=\frac{1}{z_{35} z_{24} z_{45}}, \\
& I_{21}=\frac{1}{z_{41} z_{23}^{2}}, \quad I_{22}=\frac{1}{z_{41} z_{23} z_{31}}, \quad I_{23}=\frac{1}{z_{41} z_{23} z_{35}}, \quad I_{24}=\frac{1}{z_{45} z_{23}^{2}}, \\
& I_{25}=\frac{1}{z_{45} z_{23} z_{31}}, \quad I_{26}=\frac{1}{z_{45} z_{23} z_{35}}, \quad I_{27}=\frac{1}{z_{23} z_{34}^{2}}, \quad I_{28}=\frac{1}{z_{24} z_{34}^{2}}, \\
& I_{29}=\frac{1}{z_{34} z_{24}^{2}}, \quad I_{30}=\frac{1}{z_{23} z_{34} z_{41}}, \quad I_{31}=\frac{1}{z_{23} z_{34} z_{45}}, \quad I_{32}=\frac{1}{z_{24} z_{34} z_{31}}, \\
& I_{33}=\frac{1}{z_{24} z_{34} z_{35}}, \quad I_{34}=\frac{1}{z_{23}^{2} z_{24}}, \quad I_{35}=\frac{1}{z_{24}^{2} z_{23}}, \quad I_{36}=\frac{1}{z_{23}^{2} z_{34}}, \\
& I_{37}=\frac{1}{z_{25} z_{34} z_{35}}, \quad I_{38}=\frac{1}{z_{24} z_{34} z_{45}}, \quad I_{39}=\frac{1}{z_{24} z_{25} z_{35}}, \quad I_{40}=\frac{1}{z_{24} z_{25} z_{34}}, \\
& I_{41}=\frac{1}{z_{23} z_{34} z_{35}}, \quad I_{42}=\frac{1}{z_{23} z_{25} z_{45}}, \quad I_{43}=\frac{1}{z_{23} z_{25} z_{34}}, \quad I_{44}=\frac{1}{z_{23} z_{24} z_{45}}, \\
& I_{45}=\frac{1}{z_{23} z_{24} z_{35}}, \quad I_{46}=\frac{1}{z_{23} z_{24} z_{34}}, \quad I_{47}=\frac{1}{z_{23} z_{24} z_{25}}, \quad I_{48}=\frac{1}{z_{41} z_{24} z_{34}}, \\
& I_{49}=\frac{1}{z_{41} z_{23} z_{25}}, \quad I_{50}=\frac{1}{z_{21} z_{31} z_{24}}, \quad I_{51}=\frac{1}{z_{41} z_{23} z_{24}}, \quad I_{52}=\frac{1}{z_{31} z_{25} z_{34}},
\end{aligned}
$$




$$
\begin{aligned}
& I_{53}=\frac{1}{z_{31} z_{24} z_{25}}, \quad I_{54}=\frac{1}{z_{31} z_{23} z_{34}}, \quad I_{55}=\frac{1}{z_{31} z_{23} z_{24}}, \quad I_{56}=\frac{1}{z_{21} z_{31} z_{34}}, \\
& I_{57}=\frac{1}{z_{21} z_{34} z_{35}}, \quad I_{58}=\frac{1}{z_{21} z_{24} z_{35}}, \quad I_{59}=\frac{1}{z_{21} z_{24} z_{34}}, \quad I_{60}=\frac{1}{z_{21} z_{23} z_{45}}, \\
& I_{61}=\frac{1}{z_{21} z_{23} z_{34}}, \quad I_{62}=\frac{1}{z_{21} z_{23} z_{24}}, \quad I_{63}=\frac{1}{z_{21} z_{41} z_{23}} .
\end{aligned}
$$

\section{B.1. Partial fraction identities}

It is possible to express $I_{35}, \ldots, I_{63}$ in terms of $I_{1}, \ldots, I_{34}$. To see this one uses the partial fraction relations

$$
\frac{1}{z_{j i} z_{k i}}+\frac{1}{z_{j k} z_{j i}}=\frac{1}{z_{j k} z_{k i}},
$$

and multiply each one of them by appropriate factors of $\frac{1}{z_{i j}}$ to generate the following system of equations:

$$
\begin{array}{ccc}
I_{37}-I_{14}+I_{8}=0, & -I_{38}+I_{33}+I_{20}=0, & I_{39}-I_{20}+I_{8}=0, \\
I_{40}-I_{38}+I_{14}=0, & I_{41}-I_{31}+I_{26}=0, & I_{42}-I_{26}+I_{8}=0, \\
I_{43}-I_{41}+I_{37}=0, & I_{44}+I_{38}-I_{31}=0, & I_{45}-I_{41}+I_{33}=0, \\
I_{46}+I_{28}-I_{27}=0, & I_{46}-I_{36}+I_{34}=0, & -I_{46}+I_{35}+I_{29}=0, \\
I_{47}-I_{43}+I_{40}=0, & I_{47}-I_{45}+I_{39}=0, & I_{47}-I_{44}+I_{42}=0, \\
-I_{48}+I_{32}+I_{16}=0, & I_{49}-I_{23}+I_{7}=0, & I_{50}-I_{16}+I_{1}=0, \\
I_{51}+I_{48}-I_{30}=0, & I_{52}-I_{13}+I_{5}=0, & I_{53}-I_{17}+I_{6}=0, \\
I_{54}-I_{30}+I_{22}=0, & I_{55}-I_{54}+I_{32}=0, & I_{56}-I_{10}+I_{1}=0, \\
I_{57}-I_{11}+I_{4}=0, & I_{58}-I_{19}+I_{3}=0, & I_{59}-I_{48}+I_{10}=0, \\
I_{60}-I_{25}+I_{2}=0, & I_{61}+I_{56}-I_{54}=0, & I_{62}-I_{61}+I_{59}=0, \\
I_{62}-I_{55}+I_{50}=0, & I_{63}+I_{62}-I_{51}=0, & I_{63}-I_{22}+I_{1}=0 .
\end{array}
$$

The solution is given by

$$
\begin{aligned}
& I_{35}=I_{27}-I_{28}-I_{29}, \quad I_{36}=I_{34}+I_{27}-I_{28}, \quad I_{37}=I_{14}-I_{8}, \\
& I_{38}=I_{33}+I_{20}, \quad I_{39}=I_{20}-I_{8}, \quad I_{40}=I_{33}+I_{20}-I_{14}, \\
& I_{41}=I_{31}-I_{26}, \quad I_{42}=I_{26}-I_{8}, \quad I_{43}=I_{31}-I_{26}-I_{14}+I_{8}, \\
& I_{44}=I_{31}-I_{33}-I_{20}, \quad I_{45}=I_{31}-I_{26}-I_{33}, \quad I_{46}=I_{27}-I_{28}, \\
& I_{47}=I_{31}-I_{33}-I_{20}-I_{26}+I_{8}, \quad I_{48}=I_{32}+I_{16}, \quad I_{49}=I_{23}-I_{7}, \\
& I_{50}=I_{16}-I_{1}, \quad I_{51}=I_{30}-I_{32}-I_{16}, \quad I_{52}=I_{13}-I_{5}, \\
& I_{53}=I_{17}-I_{6}, \quad I_{54}=I_{30}-I_{22}, \quad I_{55}=I_{30}-I_{22}-I_{32}, \\
& I_{56}=I_{10}-I_{1}, \quad I_{57}=I_{11}-I_{4}, \quad I_{58}=I_{19}-I_{3}, \\
& I_{59}=I_{16}-I_{10}+I_{32}, \quad I_{60}=I_{25}-I_{2}, \quad I_{61}=I_{30}-I_{22}-I_{10}+I_{1}, \\
& I_{62}=I_{1}-I_{16}-I_{22}+I_{30}-I_{32}, \quad I_{63}=I_{22}-I_{1} .
\end{aligned}
$$




\section{B.2. Total derivative relations}

With the $S L(2, \mathbf{R})$ fixing choice of $\left(z_{1}, z_{5}, z_{6}\right)=(0,1, \infty)$, all the integrals $\mathcal{I}_{j}$ share the common factor of

$$
\begin{aligned}
M\left(z_{2}, z_{3}, z_{4}\right)= & z_{2}^{-s_{12}} z_{3}^{-s_{13}} z_{4}^{-s_{14}}\left(z_{3}-z_{2}\right)^{-s_{23}}\left(z_{4}-z_{3}\right)^{-s_{34}} \\
& \times\left(z_{4}-z_{2}\right)^{-s_{24}}\left(1-z_{2}\right)^{-s_{25}}\left(1-z_{3}\right)^{-s_{35}}\left(1-z_{4}\right)^{-s_{45}} .
\end{aligned}
$$

One can obtain additional relations among the integrals using the vanishing of

$$
\begin{aligned}
& \int d z_{2} \int d z_{3} \int d z_{4} \frac{\partial}{\partial z_{I}}\left(\frac{M}{z_{i j} z_{k l}}\right)=0, \quad i, j, k, l \neq I, I=2,3,4, \\
& \int d z_{2} \int d z_{3} \int d z_{4} \frac{\partial}{\partial z_{I}}\left(\frac{M}{z_{I j} z_{k l}}\right)=0, \quad j, k, l \neq I, I=2,3,4 .
\end{aligned}
$$

Eq. (B.7) leads to 27 equations like

$$
\begin{aligned}
& s_{12} \mathcal{I}_{1}+s_{23} \mathcal{I}_{22}+s_{24} \mathcal{I}_{16}+s_{25} \mathcal{I}_{5}=0, \\
& s_{12} \mathcal{I}_{2}+s_{23} \mathcal{I}_{25}+s_{24} \mathcal{I}_{17}+s_{25} \mathcal{I}_{6}=0,
\end{aligned}
$$

etc. Likewise, Eq. (B.8) gives rise to 18 relations,

$$
\begin{aligned}
& \left(1+s_{34}\right) \mathcal{I}_{9}=\left(s_{13}+s_{23}\right) \mathcal{I}_{1}+s_{35} \mathcal{I}_{4}-\left(s_{13}+s_{23}\right) \mathcal{I}_{10}-s_{35} \mathcal{I}_{11}-s_{23} \mathcal{I}_{22}+s_{23} \mathcal{I}_{30}, \\
& \left(1+s_{34}\right) \mathcal{I}_{12}=s_{13} \mathcal{I}_{5}+\left(s_{23}+s_{35}\right) \mathcal{I}_{8}-s_{13} \mathcal{I}_{13}-\left(s_{23}+s_{35}\right) \mathcal{I}_{14}-s_{23} \mathcal{I}_{26}+s_{23} \mathcal{I}_{31},
\end{aligned}
$$

etc. It is straightforward to show that this system of equations allows all the integrals to be rewritten in terms of a basis containing six elements in agreement with the findings of [21].

\section{B.3. Canceling the tachyon poles}

Section 3.2 discusses the double-pole integrals which by themselves introduce spurious tachyon poles. In particular, the four integrals in (3.7) are proportional to $\sim\left(1+t_{2}\right)^{-1}$ which is not at all obvious to cancel. This appendix explains the mechanisms leading to their cancellation.

Let us first of all plug in the explicit superspace expressions for the kinematic the factors in (3.7):

$$
\begin{aligned}
&\left\langle L_{233434} V_{1} V_{5} V_{6}\right\rangle \mathcal{I}_{27}+\left\langle L_{243434} V_{1} V_{5} V_{6}\right\rangle \mathcal{I}_{28}+\left\langle L_{242434} V_{1} V_{5} V_{6}\right\rangle \mathcal{I}_{29} \\
&+\left\langle L_{232334} V_{1} V_{5} V_{6}\right\rangle \mathcal{I}_{36} \\
&=\left\langle\left[\left(D_{34}\left(k^{3} \cdot A^{2}\right)-D_{24}\left(k^{2} \cdot A^{3}\right)\right)\left(1+s_{24}+s_{34}\right)\right.\right. \\
&\left.\left.+D_{23}\left(k^{2} \cdot A^{4}\right)\left(1+s_{23}+s_{34}\right)\right] V_{1} V_{5} V_{6}\right\rangle \mathcal{I}_{27} \\
&+\left\langle\left[\left(D_{24}\left(k^{4} \cdot A^{3}\right)-D_{34}\left(k^{4} \cdot A^{2}\right)\right) s_{23}-D_{23}\left(k^{3} \cdot A^{4}\right) s_{24}\right] V_{1} V_{5} V_{6}\right\rangle \mathcal{I}_{27} \\
&+\left\langle\left[\left(D_{34}\left(k^{4} \cdot A^{2}\right)-D_{23}\left(k^{2} \cdot A^{4}\right)\right)\left(1+s_{23}+s_{34}\right)\right.\right. \\
&\left.\left.+D_{24}\left(k^{2} \cdot A^{3}\right)\left(1+s_{24}+s_{34}\right)\right] V_{1} V_{5} V_{6}\right\rangle \mathcal{I}_{28} \\
&+\left\langle\left[\left(D_{23}\left(k^{3} \cdot A^{4}\right)-D_{34}\left(k^{3} \cdot A^{2}\right)\right) s_{24}-D_{24}\left(k^{4} \cdot A^{3}\right) s_{23}\right] V_{1} V_{5} V_{6}\right\rangle \mathcal{I}_{28} \\
&+\left\langle D_{24}\left[A^{3} \cdot\left(k^{2}+k^{4}\right)\right] V_{1} V_{5} V_{6}\right\rangle\left(1+s_{24}\right) \mathcal{I}_{29} \\
&-\left\langle D_{23}\left[A^{4} \cdot\left(k^{2}+k^{3}\right)\right] V_{1} V_{5} V_{6}\right\rangle\left(1+s_{23}\right) \mathcal{I}_{36} .
\end{aligned}
$$


By virtue of the total derivative equations

$$
\left(1+s_{23}\right) \mathcal{I}_{36}=s_{24}\left(\mathcal{I}_{28}-\mathcal{I}_{27}\right)+\mathcal{R}_{1}, \quad\left(1+s_{24}\right) \mathcal{I}_{29}=s_{23}\left(\mathcal{I}_{28}-\mathcal{I}_{27}\right)+\mathcal{R}_{2}
$$

where $\mathcal{R}_{1}$ and $\mathcal{R}_{2}$ denote integrals free of tachyonic poles

$$
\begin{aligned}
& \mathcal{R}_{1}=-s_{12} \mathcal{I}_{30}+s_{12} \mathcal{I}_{22}+s_{12} \mathcal{I}_{10}-s_{12} \mathcal{I}_{1}-s_{25} \mathcal{I}_{31}+s_{25} \mathcal{I}_{26}+s_{25} \mathcal{I}_{14}-s_{25} \mathcal{I}_{8}, \\
& \mathcal{R}_{2}=-s_{12} \mathcal{I}_{32}-s_{12} \mathcal{I}_{16}+s_{12} \mathcal{I}_{10}-s_{25} \mathcal{I}_{33}-s_{25} \mathcal{I}_{20}+s_{25} \mathcal{I}_{14},
\end{aligned}
$$

the RHS of (B.9) becomes

$$
\begin{aligned}
= & \left\langle\left[D_{23}\left(k^{2} \cdot A^{4}\right)-D_{24}\left(k^{2} \cdot A^{3}\right)\right] V_{1} V_{5} V_{6}\right\rangle\left(1+s_{23}+s_{24}+s_{34}\right)\left(\mathcal{I}_{27}-\mathcal{I}_{28}\right) \\
& +\left\langle D_{34}\left(k^{3} \cdot A^{2}\right) V_{1} V_{5} V_{6}\right\rangle\left[\left(1+s_{24}+s_{34}\right) \mathcal{I}_{27}-s_{24} \mathcal{I}_{28}\right] \\
& +\left\langle R_{234} V_{1} V_{5} V_{6}\right\rangle \mathcal{R}_{1}+\left\langle R_{243} V_{1} V_{5} V_{6}\right\rangle \mathcal{R}_{2} \\
& +\left\langle D_{34}\left(k^{4} \cdot A^{2}\right) V_{1} V_{5} V_{6}\right\rangle\left[\left(1+s_{23}+s_{34}\right) \mathcal{I}_{28}-s_{23} \mathcal{I}_{27}\right] .
\end{aligned}
$$

Furthermore, using

$$
\begin{aligned}
& \left(1+s_{24}+s_{34}\right) \mathcal{I}_{27}-s_{24} \mathcal{I}_{28}=s_{14} \mathcal{I}_{30}+s_{45} \mathcal{I}_{31}, \\
& \left(1+s_{23}+s_{34}\right) \mathcal{I}_{28}-s_{23} \mathcal{I}_{27}=-s_{13} \mathcal{I}_{32}-s_{35} \mathcal{I}_{33}
\end{aligned}
$$

and in particular their difference, which manifestly cancels the $\left(1+t_{2}\right)^{-1}$ tachyon pole

$$
\left(1+s_{23}+s_{24}+s_{34}\right)\left(\mathcal{I}_{27}-\mathcal{I}_{28}\right)=s_{14} \mathcal{I}_{30}+s_{45} \mathcal{I}_{31}+s_{13} \mathcal{I}_{32}+s_{35} \mathcal{I}_{33}
$$

allows (B.11) to be rewritten in terms of unproblematic single-pole integrals:

$$
\begin{aligned}
& \left\langle R_{234} V_{1} V_{5} V_{6}\right\rangle \mathcal{R}_{1}+\left\langle R_{243} V_{1} V_{5} V_{6}\right\rangle \mathcal{R}_{2} \\
& \quad+\left\langle\left[D_{34}\left(k^{3} \cdot A^{2}\right)+D_{23}\left(k^{2} \cdot A^{4}\right)-D_{24}\left(k^{2} \cdot A^{3}\right)\right] V_{1} V_{5} V_{6}\right\rangle\left(s_{14} \mathcal{I}_{30}+s_{45} \mathcal{I}_{31}\right) \\
& \quad-\left\langle\left[D_{34}\left(k^{4} \cdot A^{2}\right)-D_{23}\left(k^{2} \cdot A^{4}\right)+D_{24}\left(k^{2} \cdot A^{3}\right)\right] V_{1} V_{5} V_{6}\right\rangle\left(s_{13} \mathcal{I}_{32}+s_{35} \mathcal{I}_{33}\right) .
\end{aligned}
$$

\section{B.4. Momentum expansion of the $F_{i}$ integrals}

Here we list the momentum expansion of the $F_{i}$ integrals in our end result (1.1) for the sixpoint amplitude. The first three integrals $F_{1,2,3}$ (and their parity images) multiply superfield kinematics of type $\left\langle T_{i j} T_{k l} T_{m n}\right\rangle$. Their field-theory contribution is of order $\mathcal{O}\left(s_{i j}^{-3}\right)=\mathcal{O}\left(k^{-6}\right)$, then the first superstring corrections $\mathcal{O}\left(s_{i j}^{-1}\right)=\mathcal{O}\left(k^{-2}\right)$ and $\mathcal{O}\left(s_{i j}^{0}\right)=\mathcal{O}\left(k^{0}\right)$ are multiplied by the transcendental numbers $\zeta(2)$ and $\zeta(3)$ respectively. ${ }^{10}$ More precisely, only $F_{1}$ has a non-zero field-theory limit, and the superstring corrections $\sim \zeta(2), \zeta(3)$ have no more than two poles at the same time reflecting the fact that they represent contact interactions. $F_{3}$ is even limited to single poles.

The remaining integrals $F_{4}$ to $F_{10}$ associated with $\left\langle T_{i j k} E_{l m n}\right\rangle$ kinematics have an additional power of Mandelstam invariants in their field-theory, $\zeta(2)$ - and $\zeta(3)$-terms, in order to compensate the $s_{i j}^{-1}$ within the definition of $E_{l m n}$. Once again, we find a hierarchy in their pole structure:

10 The field-theory and $\zeta(2)$ parts of the $\mathcal{I}_{j}$ are odd in the $s_{i}, t_{i}$ whereas the $\zeta(3)$ correction is even. That is why one has to be careful about the sign convention of the Mandelstam variables. The $s_{i}, t_{i}$ in the present paper as well as Refs. [25, 27] have to be replaced by $-s_{i},-t_{i}$ for comparison with [21-23]. 
The first three integrals $F_{4}, F_{5}, F_{6}$ have two kinematic poles in their field-theory limit and mostly single poles in their $\zeta(2), \zeta(3)$ corrections. The few exceptional double poles in these higher order contributions - say $\frac{s_{4} s_{5} \zeta(2)}{s_{1} t_{1}}$ in $F_{4}$ - are decorated by numerators which cancel both poles in the associated $E_{i j k}$, e.g. $s_{4} s_{5} E_{456}=s_{5} T_{45} V_{6}+s_{4} V_{4} T_{56}$. The integrals $F_{7}, F_{8}$ have single poles only from the beginning, and $F_{9}, F_{10}$ are even completely regular and start at $\zeta(3)$.

There is an infinite tower of higher order corrections in the momenta, i.e. higher orders in $\alpha^{\prime}$, along with multi-zeta values (MZVs) which we do not display here:

$$
\begin{aligned}
& F_{1}=-\int d z_{2} \int d z_{3} \int d z_{4} \prod_{i<j}^{6} \frac{\left|z_{i j}\right|^{-s_{i j}}}{s_{6}}\left(\frac{s_{12}}{z_{21} z_{23} z_{45}}+\frac{s_{13}}{z_{23} z_{31} z_{45}}\right) \\
& =\frac{1}{s_{2} s_{4} s_{6}}+\zeta(2)\left(\frac{t_{1}}{s_{2} s_{6}}-\frac{t_{1}}{s_{2} s_{4}}-\frac{s_{5}}{s_{2} s_{6}}-\frac{t_{3}}{s_{4} s_{6}}+\frac{t_{1}}{s_{4} s_{6}}+\frac{1}{s_{4}}-\frac{s_{2}}{s_{4} s_{6}}-\frac{s_{1}}{s_{4} s_{6}}\right) \\
& +\zeta(3)\left(-\frac{t_{1}^{2}}{s_{2} s_{4}}-\frac{s_{6} t_{1}}{s_{2} s_{4}}+\frac{2 t_{1} t_{2}}{s_{2} s_{6}}+\frac{t_{1}^{2}}{s_{2} s_{6}}-\frac{s_{5}^{2}}{s_{2} s_{6}}+\frac{s_{4} t_{1}}{s_{2} s_{6}}-\frac{s_{4} s_{5}}{s_{2} s_{6}}-\frac{t_{3}^{2}}{s_{4} s_{6}}\right. \\
& +\frac{t_{1} t_{3}}{s_{4} s_{6}}+\frac{t_{1}^{2}}{s_{4} s_{6}}+\frac{t_{1}}{s_{4}}+\frac{s_{6}}{s_{4}}-\frac{2 t_{2}}{s_{6}}-\frac{2 t_{1}}{s_{6}}+\frac{2 s_{3}}{s_{6}}-\frac{2 s_{2} t_{3}}{s_{4} s_{6}} \\
& \left.+\frac{2 s_{2}}{s_{6}}-\frac{s_{2}^{2}}{s_{4} s_{6}}-\frac{s_{1} t_{3}}{s_{4} s_{6}}+\frac{s_{1}}{s_{4}}-\frac{2 s_{1} s_{2}}{s_{4} s_{6}}-\frac{s_{1}^{2}}{s_{4} s_{6}}\right)+\cdots \\
& F_{2}=-\int d z_{2} \int d z_{3} \int d z_{4} \prod_{i<j}^{6} \frac{\left|z_{i j}\right|^{-s_{i j}}}{s_{6}}\left(\frac{s_{14}}{z_{25} z_{34} z_{41}}+\frac{s_{13}}{z_{25} z_{34} z_{31}}\right) \\
& =\zeta(2)\left(-\frac{t_{2}}{s_{3} s_{6}}+\frac{s_{5}}{s_{3} s_{6}}-\frac{s_{1}}{s_{3} s_{6}}\right)+\zeta(3)\left(-\frac{t_{2} t_{3}}{s_{3} s_{6}}-\frac{t_{2}^{2}}{s_{3} s_{6}}-\frac{t_{2}}{s_{3}}+\frac{s_{5} t_{3}}{s_{3} s_{6}}+\frac{s_{5}}{s_{3}}\right. \\
& \left.+\frac{s_{5}^{2}}{s_{3} s_{6}}+\frac{t_{2}}{s_{6}}-\frac{t_{1}}{s_{6}}-\frac{s_{5}}{s_{6}}+\frac{s_{2}}{s_{6}}-\frac{s_{1} t_{3}}{s_{3} s_{6}}-\frac{2 s_{1} t_{2}}{s_{3} s_{6}}-\frac{s_{1}}{s_{3}}+\frac{2 s_{1}}{s_{6}}-\frac{s_{1}^{2}}{s_{3} s_{6}}\right)+\cdots, \\
& F_{3}=-\int d z_{2} \int d z_{3} \int d z_{4} \prod_{i<j}^{6} \frac{\left|z_{i j}\right|^{-s_{i j}}}{s_{6}}\left(\frac{s_{14}}{z_{24} z_{41} z_{35}}+\frac{s_{12}}{z_{24} z_{21} z_{35}}\right) \\
& =\frac{\zeta(2)}{s_{6}}+\zeta(3)\left(\frac{t_{3}}{s_{6}}+\frac{2 t_{2}}{s_{6}}+\frac{3 t_{1}}{s_{6}}-\frac{s_{5}}{s_{6}}+\frac{s_{4}}{s_{6}}+\frac{s_{3}}{s_{6}}-\frac{2 s_{2}}{s_{6}}-\frac{2 s_{1}}{s_{6}}\right)+\cdots, \\
& F_{4}=\int d z_{2} \int d z_{3} \int d z_{4} \prod_{i<j}^{6}\left|z_{i j}\right|^{-s_{i j}} \frac{s_{45}}{z_{21} z_{31} z_{45}} \\
& =\frac{1}{s_{1} t_{1}}+\zeta(2)\left(\frac{s_{4}}{s_{1}}-\frac{t_{3}}{s_{1}}-\frac{s_{4} s_{5}}{s_{1} t_{1}}-\frac{s_{2}}{t_{1}}\right)+\zeta(3)\left(\frac{s_{4} t_{1}}{s_{1}}-\frac{t_{3}^{2}}{s_{1}}-\frac{t_{1} t_{3}}{s_{1}}-\frac{s_{4} s_{5}^{2}}{s_{1} t_{1}}\right. \\
& \left.+\frac{s_{4}^{2}}{s_{1}}-\frac{s_{4}^{2} s_{5}}{s_{1} t_{1}}+\frac{2 s_{3} s_{4}}{s_{1}}-\frac{s_{2}^{2}}{t_{1}}-\frac{s_{1} s_{2}}{t_{1}}-s_{2}+s_{6}-t_{3}\right)+\cdots, \\
& F_{5}=\int d z_{2} \int d z_{3} \int d z_{4} \prod_{i<j}^{6}\left|z_{i j}\right|^{-s_{i j}} \frac{1}{z_{23} z_{24}}\left(\frac{s_{12}}{z_{21}}+\frac{s_{13}}{z_{31}}+\frac{s_{14}}{z_{41}}\right) \\
& =-\frac{1}{s_{2} t_{2}}+\zeta(2)\left(\frac{t_{2}}{s_{2}}+\frac{t_{1}}{s_{2}}-1-\frac{s_{6}}{s_{2}}-\frac{s_{5}}{s_{2}}+\frac{s_{5} s_{6}}{s_{2} t_{2}}+\frac{s_{3}}{t_{2}}+\frac{s_{4}}{s_{2}}\right)
\end{aligned}
$$




$$
\begin{aligned}
& +\zeta(3)\left(\frac{t_{1}^{2}}{s_{2}}+\frac{2 t_{1} t_{2}}{s_{2}}+\frac{t_{2}^{2}}{s_{2}}-\frac{s_{5}^{2}}{s_{2}}-\frac{s_{6} t_{1}}{s_{2}}-\frac{s_{5} s_{6}}{s_{2}}+\frac{s_{5}^{2} s_{6}}{s_{2} t_{2}}-\frac{s_{6}^{2}}{s_{2}}+\frac{s_{5} s_{6}^{2}}{s_{2} t_{2}}-2 t_{2}\right. \\
& \left.-t_{3}+s_{6}-2 s_{1}-s_{2}+s_{3}+\frac{s_{2} s_{3}}{t_{2}}+\frac{s_{3}^{2}}{t_{2}}+\frac{s_{4} t_{1}}{s_{2}}+\frac{2 s_{4} t_{2}}{s_{2}}-\frac{s_{4} s_{5}}{s_{2}}+\frac{s_{4}^{2}}{s_{2}}\right)+\cdots, \\
& F_{6}=\int d z_{2} \int d z_{3} \int d z_{4} \prod_{i<j}^{6}\left|z_{i j}\right|^{-s_{i j}} \frac{s_{12}}{z_{21} z_{34} z_{35}} \\
& =-\frac{1}{s_{3} t_{3}}+\zeta(2)\left(\frac{s_{5}}{s_{3}}-\frac{s_{1}}{s_{3}}+\frac{s_{1} s_{6}}{s_{3} t_{3}}+\frac{s_{4}}{t_{3}}-1\right)+\zeta(3)\left(\frac{s_{5} t_{3}}{s_{3}}+\frac{s_{5}^{2}}{s_{3}}-\frac{2 s_{1} t_{2}}{s_{3}}\right. \\
& \left.-\frac{s_{1} t_{3}}{s_{3}}+\frac{s_{1} s_{6}^{2}}{s_{3} t_{3}}-\frac{s_{1}^{2}}{s_{3}}+\frac{s_{1}^{2} s_{6}}{s_{3} t_{3}}-2 t_{1}-t_{3}+2 s_{1}-s_{3}+\frac{s_{3} s_{4}}{t_{3}}+\frac{s_{4}^{2}}{t_{3}}\right)+\cdots, \\
& F_{7}=\int d z_{2} \int d z_{3} \int d z_{4} \prod_{i<j}^{6}\left|z_{i j}\right|^{-s_{i j}} \frac{s_{14}}{z_{23} z_{25} z_{41}} \\
& =-\zeta(2) \frac{s_{14}}{s_{2}}-\zeta(3) \frac{s_{14}}{s_{2}}\left(t_{1}+t_{2}+s_{4}+s_{5}+s_{6}\right)+\zeta(3) s_{14}+\cdots, \\
& F_{8}=\int d z_{2} \int d z_{3} \int d z_{4} \prod_{i<j}^{6}\left|z_{i j}\right|^{-s_{i j}} \frac{s_{35}}{z_{21} z_{35} z_{41}} \\
& =\zeta(2) \frac{s_{35}}{s_{1}}+\zeta(3) \frac{s_{35}}{s_{1}}\left(t_{1}+t_{3}+s_{3}+s_{4}+s_{5}\right)+\cdots, \\
& F_{9}=\int d z_{2} \int d z_{3} \int d z_{4} \prod_{i<j}^{6}\left|z_{i j}\right|^{-s_{i j}} \frac{s_{14}}{z_{25} z_{35} z_{41}}=\zeta(3) s_{14}+\cdots, \\
& F_{10}=\int d z_{2} \int d z_{3} \int d z_{4} \prod_{i<j}^{6}\left|z_{i j}\right|^{-s_{i j}} \frac{s_{35}}{z_{24} z_{35} z_{41}}=2 \zeta(3) s_{35}+\cdots \text {. }
\end{aligned}
$$

\section{Appendix C. Six-point subamplitudes and extended BCJ relations}

In this appendix we display the parametrization of the remaining 21 partial subamplitudes:

$$
\begin{aligned}
A(1,2,4,3,5,6)= & -\frac{n_{1}(2345)}{s_{12} s_{34} s_{56}}+\frac{n_{2}(2435)}{s_{24} s_{35} s_{124}}+\frac{n_{3}(2435)}{s_{12} s_{35} s_{124}}+\frac{n_{4}(2435)}{s_{12} s_{56} s_{124}}+\frac{n_{5}(2435)}{s_{24} s_{56} s_{124}} \\
& +\frac{n_{6}(2435)}{s_{24} s_{56} s_{156}}-\frac{n_{7}(2345)}{s_{34} s_{56} s_{156}}+\frac{n_{1}^{\prime}(2435)}{s_{16} s_{24} s_{35}}-\frac{n_{2}^{\prime}(2345)}{s_{12} s_{34} s_{126}}-\frac{n_{2}^{\prime}(2354)}{s_{12} s_{35} s_{126}} \\
& -\frac{n_{5}^{\prime}(2345)}{s_{16} s_{34} s_{126}}-\frac{n_{5}^{\prime}(2354)}{s_{16} s_{35} s_{126}}-\frac{n_{6}^{\prime}(2345)}{s_{16} s_{34} s_{156}}+\frac{n_{7}^{\prime}(2435)}{s_{16} s_{24} s_{156}}, \\
A(1,2,4,5,3,6)= & \frac{n_{1}(2453)}{s_{12} s_{36} s_{45}}-\frac{n_{2}(2435)}{s_{24} s_{35} s_{124}}-\frac{n_{3}(2435)}{s_{12} s_{35} s_{124}}+\frac{n_{4}(2453)}{s_{12} s_{36} s_{124}}+\frac{n_{5}(2453)}{s_{24} s_{36} s_{124}} \\
& +\frac{n_{6}(2453)}{s_{24} s_{36} s_{136}}+\frac{n_{7}(2453)}{s_{36} s_{45} s_{136}}-\frac{n_{1}^{\prime}(2435)}{s_{16} s_{24} s_{35}}+\frac{n_{2}^{\prime}(2354)}{s_{12} s_{35} s_{126}}-\frac{n_{3}^{\prime}(2345)}{s_{12} s_{45} s_{126}} \\
& -\frac{n_{4}^{\prime}(2345)}{s_{16} s_{45} s_{126}}+\frac{n_{5}^{\prime}(2354)}{s_{16} s_{35} s_{126}}+\frac{n_{6}^{\prime}(2453)}{s_{16} s_{45} s_{136}}+\frac{n_{7}^{\prime}(2453)}{s_{16} s_{24} s_{136}},
\end{aligned}
$$




$$
\begin{aligned}
& A(1,2,5,3,4,6)=-\frac{n_{1}(2354)}{s_{12} s_{35} s_{46}}+\frac{n_{2}(2534)}{s_{25} s_{34} s_{125}}+\frac{n_{3}(2534)}{s_{12} s_{34} s_{125}}+\frac{n_{4}(2534)}{s_{12} s_{46} s_{125}}+\frac{n_{5}(2534)}{s_{25} s_{46} s_{125}} \\
& +\frac{n_{6}(2534)}{s_{25} s_{46} s_{146}}-\frac{n_{7}(2354)}{s_{35} s_{46} s_{146}}+\frac{n_{1}^{\prime}(2534)}{s_{16} s_{25} s_{34}}-\frac{n_{2}^{\prime}(2345)}{s_{12} s_{34} s_{126}}-\frac{n_{2}^{\prime}(2354)}{s_{12} s_{35} s_{126}} \\
& -\frac{n_{5}^{\prime}(2345)}{s_{16} s_{34} s_{126}}-\frac{n_{5}^{\prime}(2354)}{s_{16} s_{35} s_{126}}-\frac{n_{6}^{\prime}(2354)}{s_{16} s_{35} s_{146}}+\frac{n_{7}^{\prime}(2534)}{s_{16} s_{25} s_{146}} \\
& A(1,2,5,4,3,6)=-\frac{n_{1}(2453)}{s_{12} s_{36} s_{45}}-\frac{n_{2}(2534)}{s_{25} s_{34} s_{125}}-\frac{n_{3}(2534)}{s_{12} s_{34} s_{125}}+\frac{n_{4}(2543)}{s_{12} s_{36} s_{125}}+\frac{n_{5}(2543)}{s_{25} s_{36} s_{125}} \\
& +\frac{n_{6}(2543)}{s_{25} s_{36} s_{136}}-\frac{n_{7}(2453)}{s_{36} s_{45} s_{136}}-\frac{n_{1}^{\prime}(2534)}{s_{16} s_{25} s_{34}}+\frac{n_{2}^{\prime}(2345)}{s_{12} s_{34} s_{126}}+\frac{n_{3}^{\prime}(2345)}{s_{12} s_{45} s_{126}} \\
& +\frac{n_{4}^{\prime}(2345)}{s_{16} s_{45} s_{126}}+\frac{n_{5}^{\prime}(2345)}{s_{16} s_{34} s_{126}}-\frac{n_{6}^{\prime}(2453)}{s_{16} s_{45} s_{136}}+\frac{n_{7}^{\prime}(2543)}{s_{16} s_{25} s_{136}} \\
& A(1,3,2,4,5,6)=\frac{n_{1}(3245)}{s_{13} s_{24} s_{56}}-\frac{n_{2}(2345)}{s_{23} s_{45} s_{123}}+\frac{n_{3}(3245)}{s_{13} s_{45} s_{123}}+\frac{n_{4}(3245)}{s_{13} s_{56} s_{123}}-\frac{n_{5}(2345)}{s_{23} s_{56} s_{123}} \\
& -\frac{n_{6}(2345)}{s_{23} s_{56} s_{156}}-\frac{n_{6}(2435)}{s_{24} s_{56} s_{156}}-\frac{n_{1}^{\prime}(2345)}{s_{16} s_{23} s_{45}}+\frac{n_{2}^{\prime}(3245)}{s_{13} s_{24} s_{136}}+\frac{n_{3}^{\prime}(3245)}{s_{13} s_{45} s_{136}} \\
& -\frac{n_{6}^{\prime}(2453)}{s_{16} s_{45} s_{136}}-\frac{n_{7}^{\prime}(2345)}{s_{16} s_{23} s_{156}}-\frac{n_{7}^{\prime}(2435)}{s_{16} s_{24} s_{156}}-\frac{n_{7}^{\prime}(2453)}{s_{16} s_{24} s_{136}}, \\
& A(1,3,2,5,4,6)=\frac{n_{1}(3254)}{s_{13} s_{25} s_{46}}+\frac{n_{2}(2345)}{s_{23} s_{45} s_{123}}-\frac{n_{3}(3245)}{s_{13} s_{45} s_{123}}+\frac{n_{4}(3254)}{s_{13} s_{46} s_{123}}-\frac{n_{5}(2354)}{s_{23} s_{46} s_{123}} \\
& -\frac{n_{6}(2354)}{s_{23} s_{46} s_{146}}-\frac{n_{6}(2534)}{s_{25} s_{46} s_{146}}+\frac{n_{1}^{\prime}(2345)}{s_{16} s_{23} s_{45}}+\frac{n_{2}^{\prime}(3254)}{s_{13} s_{25} s_{136}}-\frac{n_{3}^{\prime}(3245)}{s_{13} s_{45} s_{136}} \\
& +\frac{n_{6}^{\prime}(2453)}{s_{16} s_{45} s_{136}}-\frac{n_{7}^{\prime}(2354)}{s_{16} s_{23} s_{146}}-\frac{n_{7}^{\prime}(2534)}{s_{16} s_{25} s_{146}}-\frac{n_{7}^{\prime}(2543)}{s_{16} s_{25} s_{136}}, \\
& A(1,3,4,2,5,6)=-\frac{n_{1}(3245)}{s_{13} s_{24} s_{56}}+\frac{n_{2}(3425)}{s_{25} s_{34} s_{134}}+\frac{n_{3}(3425)}{s_{13} s_{25} s_{134}}+\frac{n_{4}(3425)}{s_{13} s_{56} s_{134}}+\frac{n_{5}(3425)}{s_{34} s_{56} s_{134}} \\
& +\frac{n_{6}(2435)}{s_{24} s_{56} s_{156}}-\frac{n_{7}(2345)}{s_{34} s_{56} s_{156}}-\frac{n_{1}^{\prime}(2534)}{s_{16} s_{25} s_{34}}-\frac{n_{2}^{\prime}(3245)}{s_{13} s_{24} s_{136}}-\frac{n_{2}^{\prime}(3254)}{s_{13} s_{25} s_{136}} \\
& -\frac{n_{6}^{\prime}(2345)}{s_{16} s_{34} s_{156}}+\frac{n_{7}^{\prime}(2435)}{s_{16} s_{24} s_{156}}+\frac{n_{7}^{\prime}(2453)}{s_{16} s_{24} s_{136}}+\frac{n_{7}^{\prime}(2543)}{s_{16} s_{25} s_{136}} \\
& A(1,3,4,5,2,6)=\frac{n_{1}(3452)}{s_{13} s_{26} s_{45}}-\frac{n_{2}(3425)}{s_{25} s_{34} s_{134}}-\frac{n_{3}(3425)}{s_{13} s_{25} s_{134}}+\frac{n_{4}(3452)}{s_{13} s_{26} s_{134}}+\frac{n_{5}(3452)}{s_{26} s_{34} s_{134}} \\
& +\frac{n_{6}(3452)}{s_{26} s_{34} s_{126}}+\frac{n_{7}(3452)}{s_{26} s_{45} s_{126}}+\frac{n_{1}^{\prime}(2534)}{s_{16} s_{25} s_{34}}+\frac{n_{2}^{\prime}(3254)}{s_{13} s_{25} s_{136}}-\frac{n_{3}^{\prime}(3245)}{s_{13} s_{45} s_{136}} \\
& -\frac{n_{4}^{\prime}(2345)}{s_{16} s_{45} s_{126}}-\frac{n_{5}^{\prime}(2345)}{s_{16} s_{34} s_{126}}+\frac{n_{6}^{\prime}(2453)}{s_{16} s_{45} s_{136}}-\frac{n_{7}^{\prime}(2543)}{s_{16} s_{25} s_{136}} \\
& A(1,3,5,2,4,6)=-\frac{n_{1}(3254)}{s_{13} s_{25} s_{46}}+\frac{n_{2}(3524)}{s_{24} s_{35} s_{135}}+\frac{n_{3}(3524)}{s_{13} s_{24} s_{135}}+\frac{n_{4}(3524)}{s_{13} s_{46} s_{135}}+\frac{n_{5}(3524)}{s_{35} s_{46} s_{135}} \\
& +\frac{n_{6}(2534)}{s_{25} s_{46} s_{146}}-\frac{n_{7}(2354)}{s_{35} s_{46} s_{146}}-\frac{n_{1}^{\prime}(2435)}{s_{16} s_{24} s_{35}}-\frac{n_{2}^{\prime}(3245)}{s_{13} s_{24} s_{136}}-\frac{n_{2}^{\prime}(3254)}{s_{13} s_{25} s_{136}}
\end{aligned}
$$




$$
\begin{aligned}
& -\frac{n_{6}^{\prime}(2354)}{s_{16} s_{35} s_{146}}+\frac{n_{7}^{\prime}(2453)}{s_{16} s_{24} s_{136}}+\frac{n_{7}^{\prime}(2534)}{s_{16} s_{25} s_{146}}+\frac{n_{7}^{\prime}(2543)}{s_{16} s_{25} s_{136}} \\
& A(1,3,5,4,2,6)=-\frac{n_{1}(3452)}{s_{13} s_{26} s_{45}}-\frac{n_{2}(3524)}{s_{24} s_{35} s_{135}}-\frac{n_{3}(3524)}{s_{13} s_{24} s_{135}}+\frac{n_{4}(3542)}{s_{13} s_{26} s_{135}}+\frac{n_{5}(3542)}{s_{26} s_{35} s_{135}} \\
& +\frac{n_{6}(3542)}{s_{26} s_{35} s_{126}}-\frac{n_{7}(3452)}{s_{26} s_{45} s_{126}}+\frac{n_{1}^{\prime}(2435)}{s_{16} s_{24} s_{35}}+\frac{n_{2}^{\prime}(3245)}{s_{13} s_{24} s_{136}}+\frac{n_{3}^{\prime}(3245)}{s_{13} s_{45} s_{136}} \\
& +\frac{n_{4}^{\prime}(2345)}{s_{16} s_{45} s_{126}}-\frac{n_{5}^{\prime}(2354)}{s_{16} s_{35} s_{126}}-\frac{n_{6}^{\prime}(2453)}{s_{16} s_{45} s_{136}}-\frac{n_{7}^{\prime}(2453)}{s_{16} s_{24} s_{136}}, \\
& A(1,4,2,3,5,6)=\frac{n_{1}(4235)}{s_{14} s_{23} s_{56}}-\frac{n_{2}(2435)}{s_{24} s_{35} s_{124}}+\frac{n_{3}(4235)}{s_{14} s_{35} s_{124}}+\frac{n_{4}(4235)}{s_{14} s_{56} s_{124}}-\frac{n_{5}(2435)}{s_{24} s_{56} s_{124}} \\
& -\frac{n_{6}(2345)}{s_{23} s_{56} s_{156}}-\frac{n_{6}(2435)}{s_{24} s_{56} s_{156}}-\frac{n_{1}^{\prime}(2435)}{s_{16} s_{24} s_{35}}+\frac{n_{2}^{\prime}(4235)}{s_{14} s_{23} s_{146}}+\frac{n_{3}^{\prime}(4235)}{s_{14} s_{35} s_{146}} \\
& -\frac{n_{6}^{\prime}(2354)}{s_{16} s_{35} s_{146}}-\frac{n_{7}^{\prime}(2345)}{s_{16} s_{23} s_{156}}-\frac{n_{7}^{\prime}(2354)}{s_{16} s_{23} s_{146}}-\frac{n_{7}^{\prime}(2435)}{s_{16} s_{24} s_{156}}, \\
& A(1,4,2,5,3,6)=\frac{n_{1}(4253)}{s_{14} s_{25} s_{36}}+\frac{n_{2}(2435)}{s_{24} s_{35} s_{124}}-\frac{n_{3}(4235)}{s_{14} s_{35} s_{124}}+\frac{n_{4}(4253)}{s_{14} s_{36} s_{124}}-\frac{n_{5}(2453)}{s_{24} s_{36} s_{124}} \\
& -\frac{n_{6}(2453)}{s_{24} s_{36} s_{136}}-\frac{n_{6}(2543)}{s_{25} s_{36} s_{136}}+\frac{n_{1}^{\prime}(2435)}{s_{16} s_{24} s_{35}}+\frac{n_{2}^{\prime}(4253)}{s_{14} s_{25} s_{146}}-\frac{n_{3}^{\prime}(4235)}{s_{14} s_{35} s_{146}} \\
& +\frac{n_{6}^{\prime}(2354)}{s_{16} s_{35} s_{146}}-\frac{n_{7}^{\prime}(2453)}{s_{16} s_{24} s_{136}}-\frac{n_{7}^{\prime}(2534)}{s_{16} s_{25} s_{146}}-\frac{n_{7}^{\prime}(2543)}{s_{16} s_{25} s_{136}}, \\
& A(1,4,3,2,5,6)=-\frac{n_{1}(4235)}{s_{14} s_{23} s_{56}}-\frac{n_{2}(3425)}{s_{25} s_{34} s_{134}}+\frac{n_{3}(4325)}{s_{14} s_{25} s_{134}}+\frac{n_{4}(4325)}{s_{14} s_{56} s_{134}}-\frac{n_{5}(3425)}{s_{34} s_{56} s_{134}} \\
& +\frac{n_{6}(2345)}{s_{23} s_{56} s_{156}}+\frac{n_{7}(2345)}{s_{34} s_{56} s_{156}}+\frac{n_{1}^{\prime}(2534)}{s_{16} s_{25} s_{34}}-\frac{n_{2}^{\prime}(4235)}{s_{14} s_{23} s_{146}}-\frac{n_{2}^{\prime}(4253)}{s_{14} s_{25} s_{146}} \\
& +\frac{n_{6}^{\prime}(2345)}{s_{16} s_{34} s_{156}}+\frac{n_{7}^{\prime}(2345)}{s_{16} s_{23} s_{156}}+\frac{n_{7}^{\prime}(2354)}{s_{16} s_{23} s_{146}}+\frac{n_{7}^{\prime}(2534)}{s_{16} s_{25} s_{146}} \\
& A(1,4,3,5,2,6)=\frac{n_{1}(4352)}{s_{14} s_{26} s_{35}}+\frac{n_{2}(3425)}{s_{25} s_{34} s_{134}}-\frac{n_{3}(4325)}{s_{14} s_{25} s_{134}}+\frac{n_{4}(4352)}{s_{14} s_{26} s_{134}}-\frac{n_{5}(3452)}{s_{26} s_{34} s_{134}} \\
& -\frac{n_{6}(3452)}{s_{26} s_{34} s_{126}}-\frac{n_{6}(3542)}{s_{26} s_{35} s_{126}}-\frac{n_{1}^{\prime}(2534)}{s_{16} s_{25} s_{34}}+\frac{n_{2}^{\prime}(4253)}{s_{14} s_{25} s_{146}}-\frac{n_{3}^{\prime}(4235)}{s_{14} s_{35} s_{146}} \\
& +\frac{n_{5}^{\prime}(2345)}{s_{16} s_{34} s_{126}}+\frac{n_{5}^{\prime}(2354)}{s_{16} s_{35} s_{126}}+\frac{n_{6}^{\prime}(2354)}{s_{16} s_{35} s_{146}}-\frac{n_{7}^{\prime}(2534)}{s_{16} s_{25} s_{146}}, \\
& A(1,4,5,2,3,6)=-\frac{n_{1}(4253)}{s_{14} s_{25} s_{36}}+\frac{n_{2}(4523)}{s_{23} s_{45} s_{145}}+\frac{n_{3}(4523)}{s_{14} s_{23} s_{145}}+\frac{n_{4}(4523)}{s_{14} s_{36} s_{145}}+\frac{n_{5}(4523)}{s_{36} s_{45} s_{145}} \\
& +\frac{n_{6}(2543)}{s_{25} s_{36} s_{136}}-\frac{n_{7}(2453)}{s_{36} s_{45} s_{136}}-\frac{n_{1}^{\prime}(2345)}{s_{16} s_{23} s_{45}}-\frac{n_{2}^{\prime}(4235)}{s_{14} s_{23} s_{146}}-\frac{n_{2}^{\prime}(4253)}{s_{14} s_{25} s_{146}} \\
& -\frac{n_{6}^{\prime}(2453)}{s_{16} s_{45} s_{136}}+\frac{n_{7}^{\prime}(2354)}{s_{16} s_{23} s_{146}}+\frac{n_{7}^{\prime}(2534)}{s_{16} s_{25} s_{146}}+\frac{n_{7}^{\prime}(2543)}{s_{16} s_{25} s_{136}} \\
& A(1,4,5,3,2,6)=-\frac{n_{1}(4352)}{s_{14} s_{26} s_{35}}-\frac{n_{2}(4523)}{s_{23} s_{45} s_{145}}-\frac{n_{3}(4523)}{s_{14} s_{23} s_{145}}+\frac{n_{4}(4532)}{s_{14} s_{26} s_{145}}+\frac{n_{5}(4532)}{s_{26} s_{45} s_{145}}
\end{aligned}
$$




$$
\begin{aligned}
& +\frac{n_{6}(3542)}{s_{26} s_{35} s_{126}}-\frac{n_{7}(3452)}{s_{26} s_{45} s_{126}}+\frac{n_{1}^{\prime}(2345)}{s_{16} s_{23} s_{45}}+\frac{n_{2}^{\prime}(4235)}{s_{14} s_{23} s_{146}}+\frac{n_{3}^{\prime}(4235)}{s_{14} s_{35} s_{146}} \\
& +\frac{n_{4}^{\prime}(2345)}{s_{16} s_{45} s_{126}}-\frac{n_{5}^{\prime}(2354)}{s_{16} s_{35} s_{126}}-\frac{n_{6}^{\prime}(2354)}{s_{16} s_{35} s_{146}}-\frac{n_{7}^{\prime}(2354)}{s_{16} s_{23} s_{146}}, \\
& A(1,5,2,3,4,6)=\frac{n_{1}(5234)}{s_{15} s_{23} s_{46}}-\frac{n_{2}(2534)}{s_{25} s_{34} s_{125}}+\frac{n_{3}(5234)}{s_{15} s_{34} s_{125}}+\frac{n_{4}(5234)}{s_{15} s_{46} s_{125}}-\frac{n_{5}(2534)}{s_{25} s_{46} s_{125}} \\
& -\frac{n_{6}(2354)}{s_{23} s_{46} s_{146}}-\frac{n_{6}(2534)}{s_{25} s_{46} s_{146}}-\frac{n_{1}^{\prime}(2534)}{s_{16} s_{25} s_{34}}+\frac{n_{2}^{\prime}(5234)}{s_{15} s_{23} s_{156}}+\frac{n_{3}^{\prime}(5234)}{s_{15} s_{34} s_{156}} \\
& -\frac{n_{6}^{\prime}(2345)}{s_{16} s_{34} s_{156}}-\frac{n_{7}^{\prime}(2345)}{s_{16} s_{23} s_{156}}-\frac{n_{7}^{\prime}(2354)}{s_{16} s_{23} s_{146}}-\frac{n_{7}^{\prime}(2534)}{s_{16} s_{25} s_{146}}, \\
& A(1,5,2,4,3,6)=\frac{n_{1}(5243)}{s_{15} s_{24} s_{36}}+\frac{n_{2}(2534)}{s_{25} s_{34} s_{125}}-\frac{n_{3}(5234)}{s_{15} s_{34} s_{125}}+\frac{n_{4}(5243)}{s_{15} s_{36} s_{125}}-\frac{n_{5}(2543)}{s_{25} s_{36} s_{125}} \\
& -\frac{n_{6}(2453)}{s_{24} s_{36} s_{136}}-\frac{n_{6}(2543)}{s_{25} s_{36} s_{136}}+\frac{n_{1}^{\prime}(2534)}{s_{16} s_{25} s_{34}}+\frac{n_{2}^{\prime}(5243)}{s_{15} s_{24} s_{156}}-\frac{n_{3}^{\prime}(5234)}{s_{15} s_{34} s_{156}} \\
& +\frac{n_{6}^{\prime}(2345)}{s_{16} s_{34} s_{156}}-\frac{n_{7}^{\prime}(2435)}{s_{16} s_{24} s_{156}}-\frac{n_{7}^{\prime}(2453)}{s_{16} s_{24} s_{136}}-\frac{n_{7}^{\prime}(2543)}{s_{16} s_{25} s_{136}}, \\
& A(1,5,3,2,4,6)=-\frac{n_{1}(5234)}{s_{15} s_{23} s_{46}}-\frac{n_{2}(3524)}{s_{24} s_{35} s_{135}}+\frac{n_{3}(5324)}{s_{15} s_{24} s_{135}}+\frac{n_{4}(5324)}{s_{15} s_{46} s_{135}}-\frac{n_{5}(3524)}{s_{35} s_{46} s_{135}} \\
& +\frac{n_{6}(2354)}{s_{23} s_{46} s_{146}}+\frac{n_{7}(2354)}{s_{35} s_{46} s_{146}}+\frac{n_{1}^{\prime}(2435)}{s_{16} s_{24} s_{35}}-\frac{n_{2}^{\prime}(5234)}{s_{15} s_{23} s_{156}}-\frac{n_{2}^{\prime}(5243)}{s_{15} s_{24} s_{156}} \\
& +\frac{n_{6}^{\prime}(2354)}{s_{16} s_{35} s_{146}}+\frac{n_{7}^{\prime}(2345)}{s_{16} s_{23} s_{156}}+\frac{n_{7}^{\prime}(2354)}{s_{16} s_{23} s_{146}}+\frac{n_{7}^{\prime}(2435)}{s_{16} s_{24} s_{156}} \\
& A(1,5,3,4,2,6)=\frac{n_{1}(5342)}{s_{15} s_{26} s_{34}}+\frac{n_{2}(3524)}{s_{24} s_{35} s_{135}}-\frac{n_{3}(5324)}{s_{15} s_{24} s_{135}}+\frac{n_{4}(5342)}{s_{15} s_{26} s_{135}}-\frac{n_{5}(3542)}{s_{26} s_{35} s_{135}} \\
& -\frac{n_{6}(3452)}{s_{26} s_{34} s_{126}}-\frac{n_{6}(3542)}{s_{26} s_{35} s_{126}}-\frac{n_{1}^{\prime}(2435)}{s_{16} s_{24} s_{35}}+\frac{n_{2}^{\prime}(5243)}{s_{15} s_{24} s_{156}}-\frac{n_{3}^{\prime}(5234)}{s_{15} s_{34} s_{156}} \\
& +\frac{n_{5}^{\prime}(2345)}{s_{16} s_{34} s_{126}}+\frac{n_{5}^{\prime}(2354)}{s_{16} s_{35} s_{126}}+\frac{n_{6}^{\prime}(2345)}{s_{16} s_{34} s_{156}}-\frac{n_{7}^{\prime}(2435)}{s_{16} s_{24} s_{156}} \\
& A(1,5,4,2,3,6)=-\frac{n_{1}(5243)}{s_{15} s_{24} s_{36}}-\frac{n_{2}(4523)}{s_{23} s_{45} s_{145}}+\frac{n_{3}(5423)}{s_{15} s_{23} s_{145}}+\frac{n_{4}(5423)}{s_{15} s_{36} s_{145}}-\frac{n_{5}(4523)}{s_{36} s_{45} s_{145}} \\
& +\frac{n_{6}(2453)}{s_{24} s_{36} s_{136}}+\frac{n_{7}(2453)}{s_{36} s_{45} s_{136}}+\frac{n_{1}^{\prime}(2345)}{s_{16} s_{23} s_{45}}-\frac{n_{2}^{\prime}(5234)}{s_{15} s_{23} s_{156}}-\frac{n_{2}^{\prime}(5243)}{s_{15} s_{24} s_{156}} \\
& +\frac{n_{6}^{\prime}(2453)}{s_{16} s_{45} s_{136}}+\frac{n_{7}^{\prime}(2345)}{s_{16} s_{23} s_{156}}+\frac{n_{7}^{\prime}(2435)}{s_{16} s_{24} s_{156}}+\frac{n_{7}^{\prime}(2453)}{s_{16} s_{24} s_{136}} \text {. }
\end{aligned}
$$

Some aspects of the Ansatz, given in Eqs. (4.1), (4.2) and (C.1), have also been discussed in $[39,42]$. The remaining 16 extended BCJ relations $(4.5)$ read:

$$
\begin{gathered}
-\frac{X_{1}}{s_{12} s_{56}}+\frac{X_{2}}{s_{23} s_{123}}+\frac{X_{3}}{s_{12} s_{123}}+\frac{X_{4}}{s_{23} s_{56}}-\frac{X_{5}}{s_{56} s_{156}}-\frac{X_{13}}{s_{56} s_{124}}-\frac{X_{16}}{s_{15} s_{36}}+\frac{X_{17}}{s_{12} s_{36}} \\
-\frac{X_{18}}{s_{36} s_{124}}-\frac{X_{19}}{s_{12} s_{125}}+\frac{X_{22}}{s_{15} s_{23}}+\frac{X_{20}}{s_{23} s_{145}}+\frac{X_{21}}{s_{36} s_{145}}-\frac{X_{23}}{s_{15} s_{125}}-\frac{X_{24}}{s_{15} s_{156}}=0,
\end{gathered}
$$




$$
\begin{aligned}
& \frac{X_{1}}{s_{12} s_{56}}+\frac{X_{5}}{s_{56} s_{156}}+\frac{X_{7}}{s_{12} s_{126}}-\frac{X_{8}}{s_{61} s_{126}}+\frac{X_{9}}{s_{61} s_{156}}-\frac{X_{25}}{s_{12} s_{45}}-\frac{X_{26}}{s_{45} s_{123}}-\frac{X_{27}}{s_{56} s_{123}} \\
& -\frac{X_{28}}{s_{45} s_{61}}+\frac{X_{29}}{s_{12} s_{124}}-\frac{X_{30}}{s_{24} s_{56}}+\frac{X_{31}}{s_{24} s_{61}}+\frac{X_{32}}{s_{24} s_{124}}+\frac{X_{33}}{s_{24} s_{136}}+\frac{X_{34}}{s_{45} s_{136}}=0, \\
& -\frac{X_{7}}{s_{12} s_{126}}+\frac{X_{8}}{s_{61} s_{126}}+\frac{X_{19}}{s_{12} s_{125}}+\frac{X_{25}}{s_{12} s_{45}}+\frac{X_{26}}{s_{45} s_{123}}+\frac{X_{28}}{s_{45} s_{61}}-\frac{X_{34}}{s_{45} s_{136}}+\frac{X_{35}}{s_{12} s_{46}} \\
& -\frac{X_{36}}{s_{46} s_{123}}-\frac{X_{37}}{s_{46} s_{146}}+\frac{X_{38}}{s_{16} s_{146}}+\frac{X_{39}}{s_{25} s_{61}}+\frac{X_{40}}{s_{25} s_{125}}+\frac{X_{41}}{s_{25} s_{136}}-\frac{X_{42}}{s_{25} s_{46}}=0, \\
& \frac{X_{3}}{s_{12} s_{123}}-\frac{X_{19}}{s_{12} s_{125}}-\frac{X_{25}}{s_{12} s_{45}}+\frac{X_{34}}{s_{45} s_{136}}-\frac{X_{40}}{s_{25} s_{125}}-\frac{X_{41}}{s_{25} s_{136}}-\frac{X_{43}}{s_{12} s_{34}}+\frac{X_{44}}{s_{45} s_{126}} \\
& +\frac{X_{45}}{s_{34} s_{126}}-\frac{X_{46}}{s_{25} s_{34}}+\frac{X_{47}}{s_{13} s_{25}}+\frac{X_{48}}{s_{13} s_{123}}-\frac{X_{49}}{s_{13} s_{134}}-\frac{X_{50}}{s_{34} s_{134}}+\frac{X_{51}}{s_{13} s_{45}}=0, \\
& -\frac{X_{5}}{s_{56} s_{156}}-\frac{X_{9}}{s_{61} s_{156}}+\frac{X_{26}}{s_{45} s_{123}}+\frac{X_{27}}{s_{56} s_{123}}+\frac{X_{28}}{s_{45} s_{61}}+\frac{X_{44}}{s_{45} s_{126}}+\frac{X_{45}}{s_{34} s_{126}}-\frac{X_{49}}{s_{13} s_{134}} \\
& -\frac{X_{50}}{s_{34} s_{134}}+\frac{X_{51}}{s_{13} s_{45}}-\frac{X_{52}}{s_{34} s_{56}}-\frac{X_{53}}{s_{61} s_{136}}-\frac{X_{54}}{s_{34} s_{61}}+\frac{X_{55}}{s_{13} s_{56}}-\frac{X_{56}}{s_{13} s_{136}}=0, \\
& -\frac{X_{3}}{s_{12} s_{123}}+\frac{X_{10}}{s_{12} s_{35}}+\frac{X_{25}}{s_{12} s_{45}}-\frac{X_{29}}{s_{12} s_{124}}-\frac{X_{32}}{s_{24} s_{124}}-\frac{X_{33}}{s_{24} s_{136}}-\frac{X_{34}}{s_{45} s_{136}}-\frac{X_{44}}{s_{45} s_{126}} \\
& -\frac{X_{48}}{s_{13} s_{123}}-\frac{X_{51}}{s_{13} s_{45}}+\frac{X_{57}}{s_{35} s_{126}}-\frac{X_{58}}{s_{24} s_{35}}+\frac{X_{59}}{s_{13} s_{24}}-\frac{X_{60}}{s_{13} s_{135}}-\frac{X_{61}}{s_{35} s_{135}}=0, \\
& -\frac{X_{11}}{s_{35} s_{61}}-\frac{X_{26}}{s_{45} s_{123}}-\frac{X_{28}}{s_{45} s_{61}}+\frac{X_{36}}{s_{46} s_{123}}+\frac{X_{37}}{s_{46} s_{146}}-\frac{X_{38}}{s_{61} s_{146}}-\frac{X_{44}}{s_{45} s_{126}}-\frac{X_{51}}{s_{13} s_{45}} \\
& +\frac{X_{53}}{s_{61} s_{136}}+\frac{X_{56}}{s_{13} s_{136}}+\frac{X_{57}}{s_{35} s_{126}}-\frac{X_{60}}{s_{13} s_{135}}-\frac{X_{61}}{s_{35} s_{135}}-\frac{X_{62}}{s_{35} s_{46}}+\frac{X_{63}}{s_{13} s_{46}}=0, \\
& -\frac{X_{7}}{s_{12} s_{126}}+\frac{X_{8}}{s_{61} s_{126}}-\frac{X_{10}}{s_{12} s_{35}}-\frac{X_{11}}{s_{35} s_{61}}-\frac{X_{12}}{s_{35} s_{124}}-\frac{X_{14}}{s_{35} s_{146}}-\frac{X_{17}}{s_{12} s_{36}}+\frac{X_{18}}{s_{36} s_{124}} \\
& +\frac{X_{19}}{s_{12} s_{125}}+\frac{X_{39}}{s_{25} s_{61}}+\frac{X_{40}}{s_{25} s_{125}}+\frac{X_{53}}{s_{61} s_{136}}+\frac{X_{77}}{s_{25} s_{36}}-\frac{X_{78}}{s_{36} s_{136}}-\frac{X_{79}}{s_{25} s_{146}}=0, \\
& \frac{X_{1}}{s_{12} s_{56}}+\frac{X_{19}}{s_{12} s_{125}}-\frac{X_{27}}{s_{56} s_{123}}+\frac{X_{29}}{s_{12} s_{124}}+\frac{X_{35}}{s_{12} s_{46}}-\frac{X_{36}}{s_{46} s_{123}}-\frac{X_{37}}{s_{46} s_{146}}+\frac{X_{40}}{s_{25} s_{125}} \\
& -\frac{X_{42}}{s_{25} s_{46}}-\frac{X_{66}}{s_{14} s_{124}}+\frac{X_{80}}{s_{25} s_{134}}-\frac{X_{81}}{s_{14} s_{56}}-\frac{X_{82}}{s_{56} s_{134}}+\frac{X_{83}}{s_{14} s_{25}}+\frac{X_{84}}{s_{14} s_{146}}=0, \\
& -\frac{X_{3}}{s_{12} s_{123}}-\frac{X_{17}}{s_{12} s_{36}}-\frac{X_{29}}{s_{12} s_{124}}-\frac{X_{32}}{s_{24} s_{124}}-\frac{X_{35}}{s_{12} s_{46}}-\frac{X_{48}}{s_{13} s_{123}}-\frac{X_{56}}{s_{13} s_{136}}+\frac{X_{59}}{s_{13} s_{24}} \\
& -\frac{X_{63}}{s_{13} s_{46}}-\frac{X_{72}}{s_{46} s_{135}}-\frac{X_{78}}{s_{36} s_{136}}-\frac{X_{85}}{s_{24} s_{135}}-\frac{X_{86}}{s_{46} s_{125}}-\frac{X_{87}}{s_{36} s_{125}}-\frac{X_{88}}{s_{24} s_{36}}=0, \\
& \frac{X_{8}}{s_{61} s_{126}}-\frac{X_{11}}{s_{35} s_{61}}-\frac{X_{14}}{s_{35} s_{146}}+\frac{X_{39}}{s_{25} s_{61}}+\frac{X_{47}}{s_{13} s_{25}}+\frac{X_{53}}{s_{61} s_{136}}+\frac{X_{56}}{s_{13} s_{136}}-\frac{X_{60}}{s_{13} s_{135}} \\
& -\frac{X_{61}}{s_{35} s_{135}}+\frac{X_{71}}{s_{26} s_{126}}-\frac{X_{79}}{s_{25} s_{146}}-\frac{X_{80}}{s_{25} s_{134}}+\frac{X_{89}}{s_{26} s_{134}}+\frac{X_{90}}{s_{26} s_{35}}-\frac{X_{91}}{s_{13} s_{26}}=0, \\
& \frac{X_{2}}{s_{23} s_{123}}+\frac{X_{6}}{s_{23} s_{61}}+\frac{X_{31}}{s_{24} s_{61}}-\frac{X_{37}}{s_{46} s_{146}}+\frac{X_{38}}{s_{61} s_{146}}-\frac{X_{48}}{s_{13} s_{123}}-\frac{X_{53}}{s_{61} s_{136}}-\frac{X_{56}}{s_{13} s_{136}}
\end{aligned}
$$




$$
\begin{gathered}
+\frac{X_{59}}{s_{13} s_{24}}-\frac{X_{63}}{s_{13} s_{46}}-\frac{X_{72}}{s_{46} s_{135}}-\frac{X_{85}}{s_{24} s_{135}}-\frac{X_{92}}{s_{23} s_{46}}-\frac{X_{93}}{s_{23} s_{156}}-\frac{X_{94}}{s_{24} s_{156}}=0, \\
\frac{X_{11}}{s_{35} s_{61}}+\frac{X_{12}}{s_{35} s_{124}}-\frac{X_{18}}{s_{36} s_{124}}+\frac{X_{28}}{s_{45} s_{61}}+\frac{X_{38}}{s_{61} s_{146}}+\frac{X_{44}}{s_{45} s_{126}}-\frac{X_{53}}{s_{61} s_{136}}-\frac{X_{57}}{s_{35} s_{126}} \\
+\frac{X_{67}}{s_{14} s_{35}}+\frac{X_{68}}{s_{14} s_{145}}+\frac{X_{69}}{s_{45} s_{145}}+\frac{X_{78}}{s_{36} s_{136}}-\frac{X_{84}}{s_{14} s_{146}}-\frac{X_{95}}{s_{14} s_{36}}+\frac{X_{96}}{s_{36} s_{45}}=0, \\
\frac{X_{2}}{s_{23} s_{123}}+\frac{X_{3}}{s_{12} s_{123}}-\frac{X_{10}}{s_{12} s_{35}}-\frac{X_{14}}{s_{35} s_{146}}+\frac{X_{15}}{s_{23} s_{146}}-\frac{X_{25}}{s_{12} s_{45}}+\frac{X_{29}}{s_{12} s_{124}}+\frac{X_{44}}{s_{45} s_{126}} \\
-\frac{X_{57}}{s_{35} s_{126}}+\frac{X_{64}}{s_{23} s_{45}}+\frac{X_{65}}{s_{14} s_{23}}-\frac{X_{66}}{s_{14} s_{124}}+\frac{X_{67}}{s_{14} s_{35}}+\frac{X_{68}}{s_{14} s_{145}}+\frac{X_{69}}{s_{45} s_{145}}=0, \\
-\frac{X_{7}}{s_{12} s_{126}}+\frac{X_{19}}{s_{12} s_{125}}+\frac{X_{23}}{s_{15} s_{125}}+\frac{X_{25}}{s_{12} s_{45}}+\frac{X_{26}}{s_{45} s_{123}}+\frac{X_{35}}{s_{12} s_{46}}-\frac{X_{36}}{s_{46} s_{123}}-\frac{X_{69}}{s_{45} s_{145}} \\
+\frac{X_{70}}{s_{26} s_{45}}-\frac{X_{71}}{s_{26} s_{126}}+\frac{X_{72}}{s_{46} s_{135}}-\frac{X_{73}}{s_{26} s_{135}}-\frac{X_{74}}{s_{15} s_{46}}+\frac{X_{75}}{s_{15} s_{26}}-\frac{X_{76}}{s_{15} s_{145}}=0, \\
X_{2}+\frac{X_{3}}{s_{12} s_{123}}+\frac{X_{6}}{s_{23} s_{61}}-\frac{X_{7}}{s_{12} s_{126}}+\frac{X_{8}}{s_{61} s_{126}}+\frac{X_{35}}{s_{12} s_{46}}-\frac{X_{37}}{s_{46} s_{146}}+\frac{X_{38}}{s_{61} s_{146}} \\
-\frac{X_{43}}{s_{12} s_{34}}+\frac{X_{54}}{s_{34} s_{61}}+\frac{X_{86}}{s_{46} s_{125}}-\frac{X_{92}}{s_{23} s_{46}}-\frac{X_{93}}{s_{23} s_{156}}+\frac{X_{97}}{s_{34} s_{125}}-\frac{X_{98}}{s_{34} s_{234}}=0 .
\end{gathered}
$$

Above, the one hundred numerator triplets $X_{i}$ are defined as follows:

$$
\begin{aligned}
& X_{1}=n_{1}(2345)-n_{4}(2345)+n_{4}(2435), \\
& X_{2}=n_{2}(2345)-n_{5}(2345)+n_{5}(2354), \\
& X_{3}=n_{3}(2345)-n_{4}(2345)+n_{4}(2354), \\
& X_{4}=-n_{1}(4235)+n_{5}(2345)-n_{6}(2345), \\
& X_{5}=-n_{6}(2345)+n_{6}(2435)+n_{7}(2345), \\
& X_{6}=n_{1}^{\prime}(2345)-n_{7}^{\prime}(2345)+n_{7}^{\prime}(2354), \\
& X_{7}=n_{2}^{\prime}(2345)-n_{2}^{\prime}(2354)-n_{3}^{\prime}(2345), \\
& X_{8}=n_{4}^{\prime}(2345)-n_{5}^{\prime}(2345)+n_{5}^{\prime}(2354), \\
& X_{9}=n_{6}^{\prime}(2345)-n_{7}^{\prime}(2345)+n_{7}^{\prime}(2435), \\
& X_{10}=-n_{1}(2354)+n_{3}(2435)+n_{2}^{\prime}(2354), \\
& X_{11}=n_{1}^{\prime}(2435)+n_{5}^{\prime}(2354)-n_{6}^{\prime}(2354), \\
& X_{12}=n_{2}(2435)-n_{3}(2435)+n_{3}(4235), \\
& X_{13}=-n_{4}(2435)+n_{4}(4235)+n_{5}(2435), \\
& X_{14}=-n_{7}(2354)+n_{3}^{\prime}(4235)+n_{6}^{\prime}(2354), \\
& X_{15}=n_{6}(2354)-n_{2}^{\prime}(4235)-n_{7}^{\prime}(2354), \\
& X_{16}=n_{1}(5243)-n_{4}(5243)+n_{4}(5423), \\
& X_{17}=n_{1}(2453)-n_{4}(2453)+n_{4}(2543), \\
& X_{18}=-n_{4}(2453)+n_{4}(4253)+n_{5}(2453), \\
& X_{19}=n_{3}(2534)-n_{4}(2534)+n_{4}(2543),
\end{aligned}
$$




$$
\begin{aligned}
& X_{20}=n_{2}(4523)-n_{3}(4523)+n_{3}(5423), \\
& X_{21}=-n_{4}(4523)+n_{4}(5423)+n_{5}(4523), \\
& X_{22}=n_{1}(5234)-n_{3}(5423)-n_{2}^{\prime}(5234), \\
& X_{23}=n_{3}(5234)-n_{4}(5234)+n_{4}(5243), \\
& X_{24}=-n_{2}^{\prime}(5234)+n_{2}^{\prime}(5243)+n_{3}^{\prime}(5234), \\
& X_{25}=-n_{1}(2453)+n_{3}(2345)-n_{3}^{\prime}(2345), \\
& X_{26}=n_{2}(2345)-n_{3}(2345)+n_{3}(3245), \\
& X_{27}=-n_{4}(2345)+n_{4}(3245)+n_{5}(2345), \\
& X_{28}=n_{1}^{\prime}(2345)-n_{4}^{\prime}(2345)-n_{6}^{\prime}(2453), \\
& X_{29}=n_{3}(2435)-n_{4}(2435)+n_{4}(2453), \\
& X_{30}=n_{1}(3245)-n_{5}(2435)+n_{6}(2435), \\
& X_{31}=n_{1}^{\prime}(2435)-n_{7}^{\prime}(2435)+n_{7}^{\prime}(2453), \\
& X_{32}=n_{2}(2435)-n_{5}(2435)+n_{5}(2453), \\
& X_{33}=n_{6}(2453)-n_{2}^{\prime}(3245)-n_{7}^{\prime}(2453), \\
& X_{34}=n_{7}(2453)-n_{3}^{\prime}(3245)-n_{6}^{\prime}(2453), \\
& X_{35}=n_{1}(2354)-n_{4}(2354)+n_{4}(2534), \\
& X_{53}=-n_{4}(2354)+n_{4}(3254)+n_{5}(2354), \\
& X_{53}=-n_{1}(3452)-n_{3}(2453)+n_{7}^{\prime}(2453)-n_{7}^{\prime}(2543), \\
& X_{37}=n_{6}(2354)-n_{6}(2534)-n_{7}(2354), \\
& X_{38}=n_{6}^{\prime}(2354)-n_{7}^{\prime}(2354)+n_{7}^{\prime}(2534), \\
& X_{39}=n_{1}^{\prime}(2534)-n_{7}^{\prime}(2534)+n_{7}^{\prime}(2543), \\
& X_{40}=n_{2}(2534)-n_{5}(2534)+n_{5}(2543), \\
& X_{41}=n_{6}(2543)-n_{2}^{\prime}(3254)-n_{7}^{\prime}(2543), \\
& X_{42}=n_{1}(3254)-n_{5}(2534)+n_{6}(2534), \\
& X_{43}=n_{1}(2345)-n_{3}(2534)-n_{2}^{\prime}(2345), \\
& X_{44}=n_{7}(3452)-n_{3}^{\prime}(2345)+n_{4}^{\prime}(2345), \\
& X_{5}(3452)-n_{2}^{\prime}(2345)+n_{5}^{\prime}(2345),
\end{aligned}
$$




$$
\begin{aligned}
& X_{55}=n_{1}(3245)-n_{4}(3245)+n_{4}(3425) \text {, } \\
& X_{56}=-n_{2}^{\prime}(3245)+n_{2}^{\prime}(3254)+n_{3}^{\prime}(3245) \text {, } \\
& X_{57}=n_{6}(3542)-n_{2}^{\prime}(2354)+n_{5}^{\prime}(2354) \text {, } \\
& X_{58}=-n_{2}(2435)+n_{2}(3524)+n_{1}^{\prime}(2435) \text {, } \\
& X_{59}=n_{1}(3245)-n_{3}(3524)-n_{2}^{\prime}(3245) \text {, } \\
& X_{60}=-n_{3}(3524)+n_{4}(3524)-n_{4}(3542) \text {, } \\
& X_{61}=-n_{2}(3524)+n_{5}(3524)-n_{5}(3542) \text {, } \\
& X_{62}=n_{1}(2354)-n_{5}(3524)-n_{7}(2354) \text {, } \\
& X_{63}=n_{1}(3254)-n_{4}(3254)+n_{4}(3524) \text {, } \\
& X_{64}=-n_{2}(2345)+n_{2}(4523)+n_{1}^{\prime}(2345) \text {, } \\
& X_{65}=-n_{1}(4235)+n_{3}(4523)+n_{2}^{\prime}(4235) \text {, } \\
& X_{66}=-n_{3}(4235)+n_{4}(4235)-n_{4}(4253) \text {, } \\
& X_{67}=n_{1}(4352)-n_{3}(4235)+n_{3}^{\prime}(4235) \text {, } \\
& X_{68}=-n_{3}(4523)+n_{4}(4523)-n_{4}(4532) \text {, } \\
& X_{69}=-n_{2}(4523)+n_{5}(4523)-n_{5}(4532) \text {, } \\
& X_{70}=n_{1}(3452)-n_{5}(4532)-n_{7}(3452) \text {, } \\
& X_{71}=n_{6}(3452)-n_{6}(3542)-n_{7}(3452) \text {, } \\
& X_{72}=-n_{4}(3524)+n_{4}(5324)+n_{5}(3524) \text {, } \\
& X_{73}=n_{4}(3542)-n_{4}(5342)-n_{5}(3542) \text {, } \\
& X_{74}=n_{1}(5234)-n_{4}(5234)+n_{4}(5324) \text {, } \\
& X_{75}=n_{1}(5342)-n_{4}(5342)+n_{4}(5432) \text {, } \\
& X_{76}=n_{3}(5423)-n_{4}(5423)+n_{4}(5432) \text {, } \\
& X_{77}=n_{1}(4253)-n_{5}(2543)+n_{6}(2543) \text {, } \\
& X_{78}=-n_{6}(2453)+n_{6}(2543)+n_{7}(2453) \text {, } \\
& X_{79}=n_{6}(2534)-n_{2}^{\prime}(4253)-n_{7}^{\prime}(2534) \text {, } \\
& X_{80}=n_{2}(3425)-n_{3}(3425)+n_{3}(4325) \text {, } \\
& X_{81}=n_{1}(4235)-n_{4}(4235)+n_{4}(4325) \text {, } \\
& X_{82}=n_{4}(3425)-n_{4}(4325)-n_{5}(3425) \text {, } \\
& X_{83}=n_{1}(4253)-n_{3}(4325)-n_{2}^{\prime}(4253) \text {, } \\
& X_{84}=-n_{2}^{\prime}(4235)+n_{2}^{\prime}(4253)+n_{3}^{\prime}(4235) \text {, } \\
& X_{85}=n_{2}(3524)-n_{3}(3524)+n_{3}(5324) \text {, } \\
& X_{86}=-n_{4}(2534)+n_{4}(5234)+n_{5}(2534) \text {, } \\
& X_{87}=-n_{4}(2543)+n_{4}(5243)+n_{5}(2543) \text {, } \\
& X_{88}=n_{1}(5243)-n_{5}(2453)+n_{6}(2453) \text {, } \\
& X_{89}=-n_{4}(3452)+n_{4}(4352)+n_{5}(3452) \text {, }
\end{aligned}
$$




$$
\begin{aligned}
& X_{90}=n_{1}(4352)-n_{5}(3542)+n_{6}(3542), \\
& X_{91}=n_{1}(3452)-n_{4}(3452)+n_{4}(3542), \\
& X_{92}=-n_{1}(5234)+n_{5}(2354)-n_{6}(2354), \\
& X_{93}=n_{6}(2345)-n_{2}^{\prime}(5234)-n_{7}^{\prime}(2345), \\
& X_{94}=n_{6}(2435)-n_{2}^{\prime}(5243)-n_{7}^{\prime}(2435), \\
& X_{95}=n_{1}(4253)-n_{4}(4253)+n_{4}(4523), \\
& X_{96}=n_{1}(2453)-n_{5}(4523)-n_{7}(2453), \\
& X_{97}=n_{2}(2534)-n_{3}(2534)+n_{3}(5234), \\
& X_{98}=n_{7}(2345)-n_{3}^{\prime}(5234)-n_{6}^{\prime}(2345), \\
& X_{99}=n_{4}(4532)-n_{4}(5432)-n_{5}(4532), \\
& X_{100}=-n_{1}(5342)+n_{5}(3452)-n_{6}(3452) .
\end{aligned}
$$

Finally, one can check that the solution to the equations $X_{i}=0$ gives rise to 81 independent relations between the 105 numerators $n_{i}$ such that they can be written in terms of the 24 independent numerators (4.12):

$$
\begin{aligned}
& m_{1}=n_{3}(2345), \quad m_{2}=n_{3}(2435), \quad m_{3}=n_{3}(3245), \quad m_{4}=n_{3}(3425), \\
& m_{5}=n_{3}(4235), \quad m_{6}=n_{3}(4325), \quad m_{7}=n_{4}(2345), \quad m_{8}=n_{4}(2435), \\
& m_{9}=n_{4}(3245), \quad m_{10}=n_{4}(3425), \quad m_{11}=n_{4}(4235), \quad m_{12}=n_{4}(4325), \\
& m_{13}=n_{2}^{\prime}(2354)=-n_{3}^{\prime}(2435), \quad m_{14}=n_{2}^{\prime}(3254)=-n_{3}^{\prime}(3425), \\
& m_{15}=n_{2}^{\prime}(4253)=-n_{3}^{\prime}(4325), \quad m_{16}=n_{3}^{\prime}(2345), \quad m_{17}=n_{3}^{\prime}(3245), \\
& m_{18}=n_{3}^{\prime}(4235), \quad m_{19}=n_{4}^{\prime}(2345), \quad m_{20}=n_{5}^{\prime}(2354)=-n_{4}^{\prime}(2435), \\
& m_{21}=n_{6}^{\prime}(2354)=-n_{4}^{\prime}(4235), \quad m_{22}=n_{6}^{\prime}(2453)=-n_{4}^{\prime}(3245), \\
& m_{23}=n_{7}^{\prime}(2435)=n_{4}^{\prime}(3425), \quad m_{24}=n_{7}^{\prime}(2534)=n_{4}^{\prime}(4325) .
\end{aligned}
$$

The equalities in (C.4) relating two different kinematic factors $n_{i}^{\prime}$ and $n_{j}^{\prime}$ follow from the symmetry properties of the BRST building blocks $T_{i j k}$ and $T_{i j k l}$. For example, to prove the identity $n_{2}^{\prime}(2354)=-n_{3}^{\prime}(2435)$ one applies the parity transformation $1 \leftrightarrow 5,2 \leftrightarrow 4$ in the kinematic factors of (4.11) to get $n_{2}^{\prime}(2345)=\left\langle\left(T_{534}-T_{543}\right) T_{21} V_{6}\right\rangle$ and $n_{3}^{\prime}(2345)=-\left\langle T_{543} T_{21} V_{6}\right\rangle$. Therefore $n_{2}^{\prime}(2354)=\left\langle\left(T_{435}-T_{453}\right) T_{21} V_{6}\right\rangle=\left\langle T_{534} T_{21} V_{6}\right\rangle=-n_{3}^{\prime}(2435)$, where the second equality follows from $T_{\{i j k\}}=T_{(i j) k}=0$. To prove $n_{6}^{\prime}(2354)=-n_{4}^{\prime}(4235)$ first use the properties $T_{i j k l}=T_{[i j] k l}, T_{[i j k] l}=T_{i j[k l]}+T_{k l[i j]}=0$ to rewrite 


$$
n_{6}(2345)=\left\langle\left(T_{1234}-T_{1324}-T_{1423}+T_{1432}\right) V_{5} V_{6}\right\rangle=-\left\langle T_{2341} V_{5} V_{6}\right\rangle
$$

which implies under parity that $n_{6}^{\prime}(2345)=-\left\langle T_{4325} V_{1} V_{6}\right\rangle$. Furthermore, the parity transformation of $n_{4}(2345)$ in (4.11) results in $n_{4}^{\prime}(2345)=\left\langle T_{5432} V_{1} V_{6}\right\rangle$ and therefore one finally obtains $n_{6}^{\prime}(2354)=-\left\langle T_{5324} V_{1} V_{6}\right\rangle=-n_{4}^{\prime}(4235)$. The other identities in (C.4) are easily shown using similar manipulations.

\section{Appendix D. The three-vertex field-theory diagrams}

Finally in this appendix we draw all 14 diagrams involving only three vertices and their corresponding pure spinor superspace expressions.
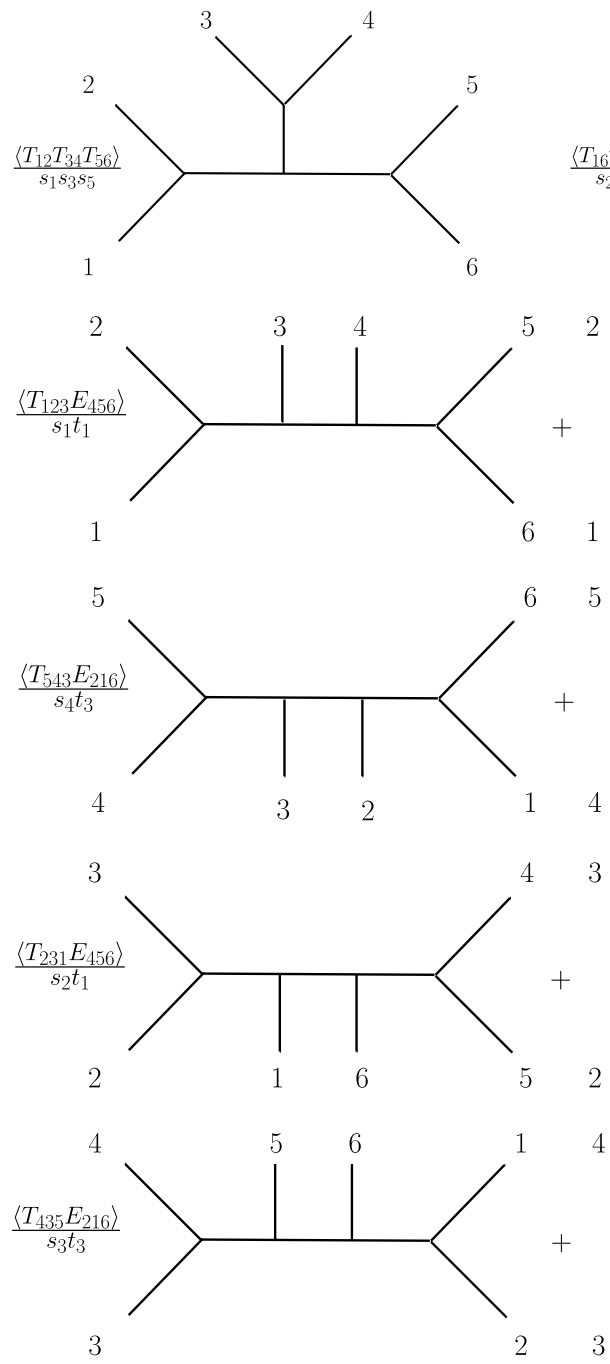

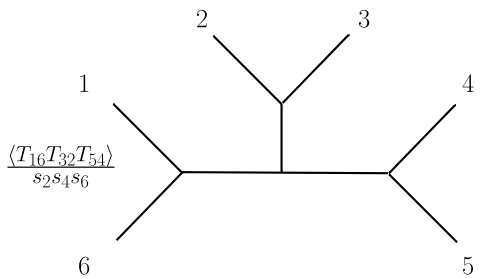

6
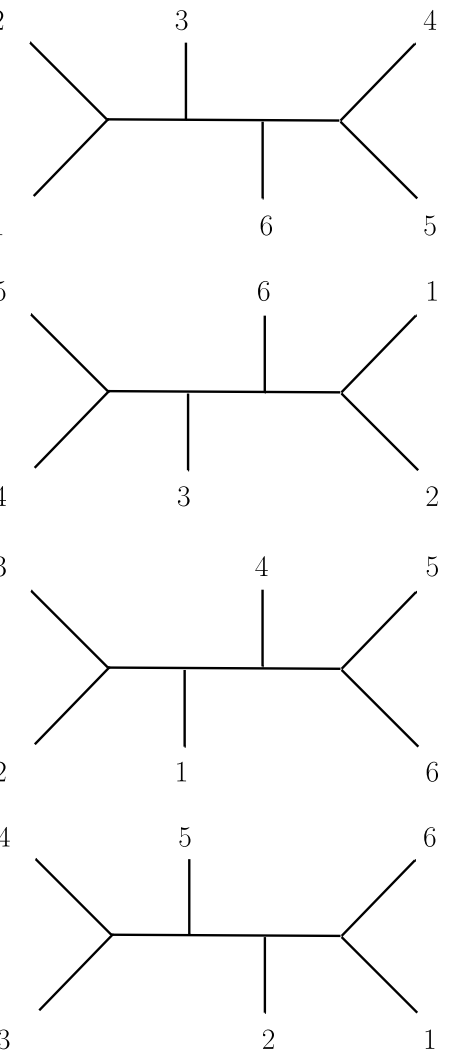

Fig. 4. The 14 field-theory diagrams and their corresponding pure spinor superspace expressions. 


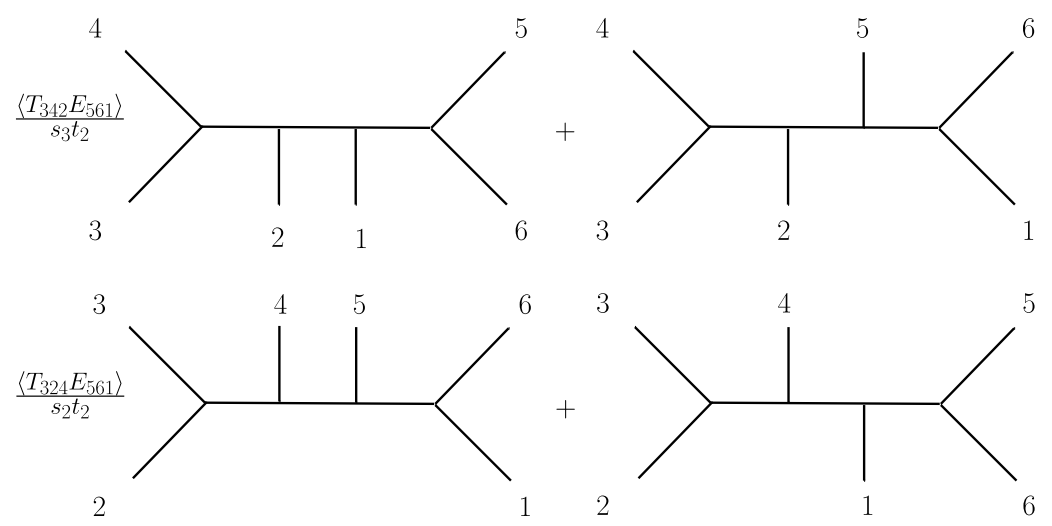

Fig. 4. Continued.

\section{References}

[1] Z. Bern, L.J. Dixon, D.A. Kosower, On-shell methods in perturbative QCD, Ann. Phys. 322 (2007) 1587, arXiv: 0704.2798 [hep-ph].

[2] Z. Bern, J.J.M. Carrasco, H. Johansson, The structure of multiloop amplitudes in gauge and gravity theories, arXiv:1007.4297 [hep-th].

[3] H. Kawai, D.C. Lewellen, S.H.H. Tye, A relation between tree amplitudes of closed and open strings, Nucl. Phys. B 269 (1986) 1.

[4] Z. Bern, J.J.M. Carrasco, H. Johansson, New relations for gauge-theory amplitudes, Phys. Rev. D 78 (2008) 085011, arXiv:0805.3993 [hep-ph].

[5] N.E.J. Bjerrum-Bohr, P.H. Damgaard, P. Vanhove, Minimal basis for gauge theory amplitudes, Phys. Rev. Lett. 103 (2009) 161602, arXiv:0907.1425 [hep-th].

[6] S. Stieberger, Open \& closed vs. pure open string disk amplitudes, arXiv:0907.2211 [hep-th].

[7] N. Berkovits, Super-Poincaré covariant quantization of the superstring, JHEP 0004 (2000) 018, arXiv:hep-th/ 0001035.

[8] N. Berkovits, Pure spinor formalism as an $N=2$ topological string, JHEP 0510 (2005) 089, arXiv:hep-th/0509120.

[9] N. Berkovits, Multiloop amplitudes and vanishing theorems using the pure spinor formalism for the superstring, JHEP 0409 (2004) 047, arXiv:hep-th/0406055.

[10] N. Berkovits, Super-Poincaré covariant two-loop superstring amplitudes, JHEP 0601 (2006) 005, arXiv:hep-th/ 0503197.

[11] N. Berkovits, N. Nekrasov, Multiloop superstring amplitudes from non-minimal pure spinor formalism, JHEP 0612 (2006) 029, arXiv:hep-th/0609012.

[12] Y. Aisaka, N. Berkovits, Pure spinor vertex operators in Siegel gauge and loop amplitude regularization, JHEP 0907 (2009) 062, arXiv:0903.3443 [hep-th].

[13] N. Berkovits, C.R. Mafra, Equivalence of two-loop superstring amplitudes in the pure spinor and RNS formalisms, Phys. Rev. Lett. 96 (2006) 011602, arXiv:hep-th/0509234.

[14] C.R. Mafra, Four-point one-loop amplitude computation in the pure spinor formalism, JHEP 0601 (2006) 075, arXiv:hep-th/0512052.

[15] C.R. Mafra, Pure spinor superspace identities for massless four-point kinematic factors, JHEP 0804 (2008) 093, arXiv:0801.0580 [hep-th].

[16] C.R. Mafra, C. Stahn, The one-loop open superstring massless five-point amplitude with the non-minimal pure spinor formalism, JHEP 0903 (2009) 126, arXiv:0902.1539 [hep-th].

[17] H. Gomez, One-loop superstring amplitude from integrals on pure spinors space, JHEP 0912 (2009) 034, arXiv:0910.3405 [hep-th].

[18] H. Gomez, C.R. Mafra, The overall coefficient of the two-loop superstring amplitude using pure spinors, JHEP 1005 (2010) 017, arXiv:1003.0678 [hep-th].

[19] N. Berkovits, B.C. Vallilo, Consistency of super-Poincaré covariant superstring tree amplitudes, JHEP 0007 (2000) 015, arXiv:hep-th/0004171. 
[20] R. Medina, F.T. Brandt, F.R. Machado, The open superstring 5-point amplitude revisited, JHEP 0207 (2002) 071 , arXiv:hep-th/0208121;

L.A. Barreiro, R. Medina, 5-field terms in the open superstring effective action, JHEP 0503 (2005) 055, arXiv:hepth/0503182.

[21] D. Oprisa, S. Stieberger, Six gluon open superstring disk amplitude, multiple hypergeometric series and EulerZagier sums, arXiv:hep-th/0509042.

[22] S. Stieberger, T.R. Taylor, Amplitude for $N$-gluon superstring scattering, Phys. Rev. Lett. 97 (2006) 211601, arXiv: hep-th/0607184;

S. Stieberger, T.R. Taylor, Multi-gluon scattering in open superstring theory, Phys. Rev. D 74 (2006) 126007, arXiv: hep-th/0609175.

[23] S. Stieberger, T.R. Taylor, Supersymmetry relations and MHV amplitudes in superstring theory, Nucl. Phys. B 793 (2008) 83, arXiv:0708.0574 [hep-th].

[24] S. Stieberger, T.R. Taylor, Complete six-gluon disk amplitude in superstring theory, Nucl. Phys. B 801 (2008) 128, arXiv:0711.4354 [hep-th].

[25] C.R. Mafra, Simplifying the tree-level superstring massless five-point amplitude, JHEP 1001 (2010) 007, arXiv: 0909.5206 [hep-th].

[26] N. Berkovits, Explaining pure spinor superspace, arXiv:hep-th/0612021.

[27] C.R. Mafra, Towards field theory amplitudes from the cohomology of pure spinor superspace, JHEP 1011 (2010) 096, arXiv:1007.3639 [hep-th].

[28] C.R. Mafra, O. Schlotterer, S. Stieberger, D. Tsimpis, A recursive formula for N-point SYM tree amplitudes, arXiv: 1012.3981 [hep-th].

[29] J.A.M. Vermaseren, New features of FORM, arXiv:math-ph/0010025;

M. Tentyukov, J.A.M. Vermaseren, The multithreaded version of FORM, arXiv:hep-ph/0702279.

[30] C.R. Mafra, PSS: A FORM program to evaluate pure spinor superspace expressions, arXiv:1007.4999 [hep-th].

[31] N. Berkovits, ICTP lectures on covariant quantization of the superstring, arXiv:hep-th/0209059.

[32] O.A. Bedoya, N. Berkovits, GGI lectures on the pure spinor formalism of the superstring, arXiv:0910.2254 [hep-th].

[33] E. Witten, Twistor-like transform in ten-dimensions, Nucl. Phys. B 266 (1986) 245.

[34] J.P. Harnad, S. Shnider, Constraints and field equations for ten-dimensional Super-Yang-Mills theory, Commun. Math. Phys. 106 (1986) 183;

H. Ooguri, J. Rahmfeld, H. Robins, J. Tannenhauser, Holography in superspace, JHEP 0007 (2000) 045, arXiv:hepth/0007104;

P.A. Grassi, L. Tamassia, Vertex operators for closed superstrings, JHEP 0407 (2004) 071, arXiv:hep-th/0405072.

[35] G. Policastro, D. Tsimpis, $R^{4}$, purified, Class. Quantum Grav. 23 (2006) 4753, arXiv:hep-th/0603165.

[36] C.R. Mafra, S. Stieberger, work in progress.

[37] R. Kleiss, H. Kuijf, Multigluon cross sections and 5-jet production at hadron colliders, Nucl. Phys. B 312 (1989) 616.

[38] V. Del Duca, L.J. Dixon, F. Maltoni, New color decompositions for gauge amplitudes at tree and loop level, Nucl. Phys. B 571 (2000) 51, arXiv:hep-ph/9910563.

[39] S.H.H. Tye, Y. Zhang, Dual identities inside the gluon and the graviton scattering amplitudes, JHEP 1006 (2010) 071, arXiv:1003.1732 [hep-th].

[40] N.E.J. Bjerrum-Bohr, P.H. Damgaard, T. Sondergaard, P. Vanhove, Monodromy and Jacobi-like relations for colorordered amplitudes, JHEP 1006 (2010) 003, arXiv:1003.2403 [hep-th].

[41] D. Binosi, L. Theussl, JaxoDraw: A graphical user interface for drawing Feynman diagrams, Comput. Phys. Commun. 161 (2004) 76, arXiv:hep-ph/0309015.

[42] D. Vaman, Y.P. Yao, Constraints and generalized gauge transformations on tree-level gluon and graviton amplitudes, arXiv:1007.3475 [hep-th]. 